\title{
Development of a Front Tracking Method for Two-Phase Micromixing of Incompressible Viscous Fluids with Interfacial Tension in Solvent Extraction*
}

\author{
Y. Zhou ${ }^{\dagger}$ N. Ray ${ }^{\dagger}$ H. Lim $†$ S. Wang \\ V. F. de Almeida $;$ J. Glimm \\ OAk Ridge National Laboratory \\ OAK RIDGE, TN 37831-6181, USA
}

Technical Report ORNL/TM-2012/28

The submitted manuscript has been authored by a contractor of the U.S. Government under the contract No. DE-AC05-00OR22725. Accordingly, the U.S. Government retains a nonexclusive, royalty-free license to publish or reproduce the published form of this contribution, or allow other to do so, for U.S. Government purposes.

13 June 2012

${ }^{\dagger}$ Dept. of Applied Mathematics and Statistics, SUNY at Stony Brook

${ }^{\ddagger}$ Oak Ridge National Laboratory

* Supported in part by the Nuclear Energy University Program of the Department of Energy, Battelle Energy Alliance LLC 00088495 (sub-award with DOE as prime sponsor), Leland Stanford Junior University 2175022040367A (sub-award with DOE as prime sponsor), Army Research Office W911NF0910306, Department of Energy DEFC0206ER25770. Computational resources were provided by the Stony Brook Galaxy cluster, the Stony Brook/BNL New York Blue Gene/L IBM machine, and the Idaho Nat. Lab. Center for Advanced Modeling and Simulation. 


\begin{abstract}
This progress report describes the development of a front tracking method for the solution of the governing equations of motion for two-phase micromixing of incompressible, viscous, liquid-liquid solvent extraction processes. The ability to compute the detailed local interfacial structure of the mixture allows characterization of the statistical properties of the two-phase mixture in terms of droplets, filaments, and other structures which emerge as a dispersed phase embedded into a continuous phase. Such a statistical picture provides the information needed for building a consistent coarsened model applicable to the entire mixing device. Coarsening is an undertaking for a future mathematical development and is outside the scope of the present work.

We present here a method for accurate simulation of the micromixing dynamics of an aqueous and an organic phase exposed to intense centrifugal force and shearing stress. The onset of mixing is the result of the combination of the classical RayleighTaylor and Kelvin-Helmholtz instabilities. A mixing environment that emulates a sector of the annular mixing zone of a centrifugal contactor is used for the mathematical domain. The domain is small enough to allow for resolution of the individual interfacial structures and large enough to allow for an analysis of their statistical distribution of sizes and shapes.

A set of accurate algorithms for this application requires an advanced front tracking approach constrained by the incompressibility condition. This research is aimed at designing and implementing these algorithms. We demonstrate verification and convergence results for one-phase and unmixed, two-phase flows. In addition we report on preliminary results for mixed, two-phase flow for realistic operating flow parameters.
\end{abstract}




\section{Contents}

List of Figures $\quad$ iv

List of Tables $\quad$ v

1 Introduction 1

2 Discontinuous equations of motion 2

2.1 Reformulated equations of motion . . . . . . . . . . . . . . 4

2.2 Continuous approximation of the equations of motion . . . . . . 5

3 Approximate solution methods $\quad 7$

3.1 Solution of the equations of motions on a fixed domain . . . . . . 8

3.2 Numerical description of fluid interfaces . . . . . . . . . . . . . . 8

3.3 Brief survey of methods for fluid flows with interfaces . . . . . . . . . 9

3.4 Front tracking with the immersed boundary method . . . . . . . . . . 11

4 Verification of one-phase flow by the method of manufactured solutions $\quad \mathbf{1 5}$

4.1 Time-dependent solutions in 2D Cartesian coordinates . . . . . . . 15

4.2 Time-dependent solutions in 3D Cartesian coordinates . . . . . . . . 16

4.3 Time-dependent solutions in 3D cylindrical coordinates . . . . . . . 18

5 Verification and validation of one-phase annular Couette flow 19

5.1 Steady-state 1-D Couette flow in an annular sector . . . . . . . . . . 22

5.2 Transition from 1-D to 3-D Taylor vortices . . . . . . . . . . . . . 22

5.3 Verifying the Couette flow perturbation growth rate . . . . . . . . 24

5.4 Turbulent Taylor-Couette flow: verification and validation . . . . . 25

6 Verification of two-phase annular Couette flow 28

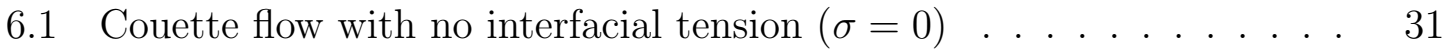

6.2 Couette flow with perturbed interfacial tension $(\sigma=0.001 \mathrm{dyn} / \mathrm{cm})$. 31

6.3 Couette flow with interfacial tension $(\sigma=10 \mathrm{dyn} / \mathrm{cm}) \quad \ldots . . . .33$

7 Two-phase Couette mixing in a 3-D annular sector 34

8 Summary and outlook $\quad 39$

A Equations of motion for incompressible Newtonian fluids $\quad 40$

A.1 One-phase fluid flow . . . . . . . . . . . . . . . . 40

A.2 Axisymmetric linear stability analysis of Couette flow . . . . . . . . . 40 
A.3 Two-phase fluid flow . . . . . . . . . . . . . . . . . . . . 45

A.4 Large-eddy filtered equations for one-phase fluid flow . . . . . . . . . 46

A.5 Code structure for incompressible fluid flow in the FronTier package . 49 


\section{List of Figures}

5.1 Left: Schematic diagram of counter-rotating axisymmetric vortices of Taylor-Couette flow (C2000, Mike Minbiole and Richard M. Lueptow). Right: Axisymmetric Taylor vortices visualized using titanium dioxidecoated mica flakes (@)2002, Alp Akonur and Richard M. Lueptow) . .

5.2 Computed velocity streamlines in the $r-z$ plane of a Taylor vortex flow at $R e=2686.3\left(\right.$ i.e. $\left.T=3.2 \times 10^{6}\right) \ldots \ldots \ldots \ldots \ldots \ldots$

5.3 Computed instability growth $\ln K$ at $R e=125$ for a Taylor-Couette flow 26

5.4 Computed instability growth $\ln K$ at $R e=200$ for a Taylor-Couette flow 26

5.5 Calculated growth rate vs. Reynolds number for a Taylor-Couette flow 27

5.6 Mean angular momentum $\left\langle v_{\theta} r\right\rangle / U_{1} R_{\mathrm{i}}$ for a turbulent (LES model) single-phase Couette flow. . . . . . . . . . . . . . . . 28

5.7 Mean azimuthal velocity $\left\langle v_{\theta}\right\rangle / U_{1}$ for a turbulent (LES model) singlephase Couette flow. . . . . . . . . . . . . . . . . . . . . . 29

7.1 Annular sector of a centrifugal contactor used for the computation domain. . . . . . . . . . . . . . . . . 35

7.2 Evolution from a "cold start" of a liquid-liquid interface in a cylindrical annular sector . . . . . . . . . . . . . . . . 37

7.3 Growth of interfacial area and conservation of volume for an initial stage of micromixing (fig. 7.2) . . . . . . . . . . . . . . 38

A.1 Neutral curve of axisymmetric, linear stability analysis (Prima and

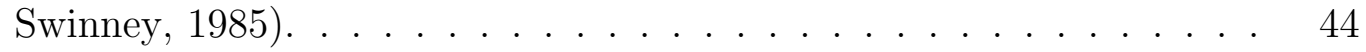

A.2 Hierarchy class structure for the incompressible solver. . . . . . . 50 


\section{List of Tables}

4.1 Verification of convergence rate of the $u$-component of the velocity for 2D NSE in Cartesian coordinates. . . . . . . . . . . . . 16

4.2 Verification of convergence rate of the $v$-component of the velocity for 2D NSE in Cartesian coordinates. . . . . . . . . . . . 16

4.3 Verification of convergence rate of the pressure $p$ for 2D NSE in Cartesian coordinates. . . . . . . . . . . . . . . . 17

4.4 Verification of convergence rate of $u$-component velocity for 3D NSE in Cartesian coordinates. . . . . . . . . . . . . . . 17

4.5 Verification of convergence rate of pressure $p$ for 3D NSE in Cartesian coordinates. . . . . . . . . . . . . . . . 18

4.6 Verification of convergence rate of $u_{\theta}$ for 3D NSE in cylindrical coordinates. . . . . . . . . . . . . . . . . . . . 19

4.7 Verification of convergence rate of $u_{z}$ for 3D NSE in cylindrical coordinates. . . . . . . . . . . . . . . . .

4.8 Verification of convergence rate of $u_{r}$ for 3D NSE in cylindrical coordinates. . . . . . . . . . . . . . . . . . . 20

4.9 Verification of convergence rate of $p$ for 3D NSE in cylindrical coordinates. . . . . . . . . . . . . . . . . . . 20

5.1 Verification of the convergence rate of $u_{\theta}$ for 1D Couette flow. . . . . 23

5.2 Verification of the convergence rate of $u_{r}$ for 1D Couette flow. . . . 23

5.3 Verification of the convergence rate of $p$ for 1D Couette flow. . . . . 23

6.1 Solution verification and calculation of the convergence rate of the interface position for 1D, two-phase Couette flow without interfacial tension, $\sigma=0 . \ldots \ldots \ldots \ldots \ldots \ldots \ldots \ldots$

6.2 Solution verification and calculation of the convergence rate of $u_{\theta}$ for 1D, two-phase Couette flow without interfacial tension, $\sigma=0$. . . .

6.3 Solution verification and calculation of the convergence rate of $p$ for 1D, two-phase Couette flow without interfacial tension, $\sigma=0$. . . .

6.4 Solution verification and calculation of the convergence rate of the interface position for 1D, two-phase Couette flow with perturbed interfacial tension, $\sigma=0.001 \mathrm{dyn} / \mathrm{cm} . \quad \ldots \ldots \ldots \ldots$. . . . .

6.5 Solution verification and calculation of the convergence rate of $u_{\theta}$ for 1D, two-phase Couette flow with perturbed interfacial tension, $\sigma=$ $0.001 \mathrm{dyn} / \mathrm{cm}$.

6.6 Solution verification and calculation of the convergence rate of $p$ for 1D, two-phase Couette flow with perturbed interfacial tension, $\sigma=$ $0.001 \mathrm{dyn} / \mathrm{cm}$. 
6.7 Solution verification and calculation of the convergence rate of the interface position for 1D, two-phase Couette flow with interfacial tension, $\sigma=10 \mathrm{dyn} / \mathrm{cm} \ldots \ldots \ldots \ldots \ldots$

6.8 Solution verification and calculation of the convergence rate of $u_{\theta}$ for 1D, two-phase Couette flow with interfacial tension, $\sigma=10 \mathrm{dyn} / \mathrm{cm}$.

6.9 Solution verification and calculation of the convergence rate of $p$ for $1 \mathrm{D}$, two-phase Couette flow with interfacial tension, $\sigma=10 \mathrm{dyn} / \mathrm{cm}$.

7.1 Geometric parameters and physical properties for 3D simulation of two-phase flow in an annular sector. . . . . . . . . . . . . 36

A.1 Critical Reynolds number $(\Gamma=\infty, \mu=0)$ of axisymmetric, linear stability analysis. . . . . . . . . . . . . . . 


\section{Introduction}

Mixing of multiple phases, as a problem in computational continuum physics, has both practical and basic scientific interest. We consider here two-phase mixtures of immiscible, incompressible, viscous liquids with non-vanishing interfacial tension. The main purpose of this report is to specify the computational framework we apply for the associated fluid flow problem, and to demonstrate verification and validation results.

A variety of methods have been proposed for solving the equations of motion for two-phase fluid flow, which generally are second-order accurate in space over the the smooth regions of the flow, i.e., away from the interface between the phases. In contrast, the interfacial discontinuity is a challenge for preserving consistent secondorder accuracy. The algorithm proposed here is second-order accurate in some of its substeps but first-order near the interfacial region.

In solvent extraction, two immiscible liquids are put in intimate contact and vigorously agitated. We are interested in the combined use of centrifugal and shear forces for mixing two incompressible liquid phases. An initially stationary interface between the phases can be greatly stretched as the fluids are mixed by these two forces.

It is unknown, both experimentally and theoretically, to what extent the specific interfacial area varies with physical properties and force strength.

The present work is aimed at shedding light into basic questions of the dynamic of mixing in this system, such as: what parameters regulate phase connectivity? What is the fully developed regime, if any? How extensive can the interfacial area be? Can volume fraction be preserved? These and other questions can only be entertained with a powerful simulation capability able to investigate the microflow length and time scales. This work is a step toward developing this capability.

In this report various efforts are organized in a progressive fashion. In Section 2, the mathematical problem is described. Section 3 presents a review of possible solution methods and a description of the algorithm proposed here, that is, a combination of the Front Tracking and Immersed Boundary methods. In Section 4, convergence and order of accuracy for our method are demonstrated for one-phase flows in both Cartesian and cylindrical coordinates. In Section 5, we focus on a one-phase version of a related problem, namely the Taylor-Couette flow in a high speed, turbulent flow regime. Accuracy and convergence specific to this flow and its geometry is considered by way of calculations of the transition from laminar flow to Taylor-Couette vortices. In addition, growth rates of perturbations slightly above the critical Taylor number are presented. Section 6 demonstrates verification of solutions for laminar two-phase flows. Section 7 presents a preliminary study of two-phase Couette mixing flow in a turbulent regime for an annular sector. The detailed equations used and overall code programming structure are presented in the Appendix subsections. 
Zhou, Ray, Lim, Wang, De Almeida, Glimm, Li, and Jiao

\section{Discontinuous equations of motion}

The incompressible Navier-Stokes equations (NSE) for a homogeneous, Newtonian fluid phase $(\alpha)$ describe a relationship between the velocity $\boldsymbol{v}_{\alpha}\left(\boldsymbol{x}_{\alpha}, t\right)$ and the hydrodynamic pressure $p_{\alpha}\left(\boldsymbol{x}_{\alpha}, t\right)$ fields at any spatial point $\boldsymbol{x}_{\alpha}$ in the bulk of a threedimensional, bounded domain $\Omega_{\alpha}(t)$ occupied by the fluid phase at the instant $t$, namely

$$
\begin{aligned}
& \rho_{\alpha} \partial_{t} \boldsymbol{v}_{\alpha}+\rho_{\alpha} \operatorname{div}_{\boldsymbol{x}}\left(\boldsymbol{v}_{\alpha} \otimes \boldsymbol{v}_{\alpha}\right)=\operatorname{div}_{\boldsymbol{x}} \boldsymbol{T}_{\alpha}+\boldsymbol{b}_{\alpha} \quad \text { in } \Omega_{\alpha}(t) \text {, and } \\
& \operatorname{div}_{\boldsymbol{x}} \boldsymbol{v}_{\alpha}=0 \quad \text { in } \Omega_{\alpha}(t),
\end{aligned}
$$

where $\boldsymbol{T}_{\alpha}\left(\boldsymbol{x}_{\alpha}, t\right)$ is the Cauchy stress tensor and $\boldsymbol{b}_{\alpha}\left(\boldsymbol{x}_{\alpha}, t\right)$ is the body force on the fluid evaluated at $\boldsymbol{x}_{\alpha} \in \Omega_{\alpha}(t)$. A fluid with homogeneous Newtonian behavior is characterized by its constitutive equation for the stress $\boldsymbol{T}_{\alpha}\left(\boldsymbol{x}_{\alpha}, t\right):=-p_{\alpha} \boldsymbol{I}+\mu_{\alpha}\left(\nabla_{\boldsymbol{x}} \boldsymbol{v}_{\alpha}+\nabla_{\boldsymbol{x}} \boldsymbol{v}_{\alpha}^{T}\right)$. Equation (2.1b) is the reduced form of the principle of mass conservation for an isochoric flow. Equation (2.1a) is the momentum balance for the fluid when incompressibility holds. In a two-phase system, another set of equations similar to (2.1) are needed for the $\beta$ phase where the immiscibility condition prompts $\Omega_{\alpha} \cap \Omega_{\beta}=\emptyset$.

The mass density $\rho_{\alpha}$ and the dynamic viscosity $\mu_{\alpha}$ are constants provided by experimental measurements. The typical body force is the result of the gravitational acceleration on the mass of the phase.

When two immiscible fluid phases $(\alpha$ and $\beta$ ) are put into contact, an inhomogeneous, three-dimensional, interfacial region between the two fluids is formed. A classical continuum approximation of this region is made by the Gibbs' dividing surface, i.e., the region is approximated by a mathematical surface $\mathcal{S}(t)=$ Clo $\Omega_{\alpha} \cap$ Clo $\Omega_{\beta}$, that is, the intersection of the closure of the domains occupied by the fluid phases. This is a justified approximation for the application of this work where water is in contact with an organic liquid (in this case a mixture of dodecane and tri- $n$-butylphosphate) and the thickness of the interfacial region is predicted to be on the order of $1 \mathrm{~nm}$ (Ye et al., 2010).

The equations (2.1) hold for each fluid phase up to the interfacial surface where a discontinuity in mass density $\rho$ and dynamic viscosity $\mu$ exists $\left(\rho_{\alpha} \neq \rho_{\beta}\right.$ and $\left.\mu_{\alpha} \neq \mu_{\beta}\right)$. The intrinsic motion of the interface is governed by a momentum and mass balance on $\mathcal{S}$, also referred to as pointwise jump conditions, respectively

$$
\begin{aligned}
& \operatorname{div}_{\mathcal{S}} \mathbb{T}+\llbracket \boldsymbol{T} \cdot \boldsymbol{n} \rrbracket=\mathbf{0} \quad \text { on } \quad \mathcal{S}(t), \quad \text { and } \\
& \llbracket(\boldsymbol{v}-\dot{\boldsymbol{x}}) \cdot \boldsymbol{n} \rrbracket=0 \quad \text { on } \quad \mathcal{S}(t),
\end{aligned}
$$

where these equations hold for the particular case of no mass transport between phases, and in the absence of any mass density excess associated to the interface. If these two conditions do not hold, then (2.2) are incomplete. The tangential surface 
stress tensor, $\mathbb{T}$, defines the interfacial state of stress while the interface velocity is denoted by $\dot{\boldsymbol{x}}$. The notation $\llbracket \cdot \rrbracket$ represents the jump across the interface of the quantity inside the brackets, that is, $\llbracket \cdot \rrbracket:=(\cdot)_{\alpha}-(\cdot)_{\beta}$ with $\boldsymbol{n}$ denoting the local unit normal vector on $\mathcal{S}$ pointing into $\alpha$, that is, $\boldsymbol{n}:=\boldsymbol{n}_{\overrightarrow{\beta \alpha}}$.

Therefore, these bracket terms couple the intrinsic motion of the interface with the neighboring phases. An interface with no intrinsic viscosity can be described by a constitutive stress restriction of the form, $\mathbb{T}:=\sigma \mathbb{I}$ with a constant interfacial tension $\sigma$. Therefore in (2.2a), the surface divergence of the interfacial stress tensor for an inviscid interface results in the familiar surface curvature term

$$
\operatorname{div}_{\mathcal{S}}(\sigma \mathbb{I})=2 \mathcal{H} \sigma \boldsymbol{n},
$$

where $\mathcal{H}$ is the local mean curvature of $\mathcal{S}$.

Modulo initial and boundary conditions, the governing equations (2.1) as applied within each continuous, homogeneous phase, coupled with the interfacial balances (2.2), describe the motion of immiscible, viscous phases in intimate contact by virtue of their interfaces. The rigorous derivation of the governing equations for the motion of fluid phases in contact through a Newtonian interface was first made by Scriven (1960) in the so called Eulerian form.

A comment on $(2.2 \mathrm{~b})$ is in order. Note that only the jump in the normal component of the velocity of the fluid relative to the interface surface velocity must vanish. This enforces no mass transfer between the phases and allows for slip of one phase past another, that is, the tangential component of the relative velocity is not constrained. This may be of significance when the fluids are non-Newtonian, high-molecular-weight polymers. If there exists slip between phases, an additional model of this phenomenon must be proposed. Therefore if no slip exists, $(2.2 \mathrm{~b})$ should be replaced by

$$
\llbracket(\boldsymbol{v}-\dot{\boldsymbol{x}}) \rrbracket=\mathbf{0} \text { on } \mathcal{S}(t),
$$

which incorporates the continuity of the phase velocities $\boldsymbol{v}_{\alpha}$ and $\boldsymbol{v}_{\beta}$ on $\mathcal{S}$.

Calculation of the three-dimensional, time evolution of $\mathcal{S}$ and the accompanying fields, $\boldsymbol{v}_{\alpha}, \boldsymbol{v}_{\beta}, p_{\alpha}, p_{\beta}$, and $\dot{\boldsymbol{x}}$, is a formidable computational task in systems involving vigorous mixing. Here the topological changes subjected by $\mathcal{S}$, lead to a dispersion with many filaments, drops, and other disconnected surface elements and phase subregions as it is typically visualized in flow mixing experiments. Hence $\mathcal{S}$ could be a highly disconnected set. A central computational difficulty is how to apply the interfacial momentum balance $(2.2 \mathrm{a})$ on $\mathcal{S}$, while resolving topological transitions which often lead to the formation of contact points, lines, surfaces, and cusps. This difficulty is exacerbated when three-dimensional vigorous mixing is present.

In order to make (2.1)-(2.2) tractable computationally, an approximation is introduced by considering the mass density and dynamic viscosity as varying in time and 
Zhou, Ray, Lim, Wang, De Almeida, Glimm, Li, and Jiao

space across the union of the domains occupied by the phases, while the divergence of the interfacial stress term is incorporated in the momentum balance as a source term in a distribution sense. This approximation is elaborated next and comments are made on the associated adverse effects.

\subsection{Reformulated equations of motion}

The interfacial equations (2.2) can be absorbed into (2.1) to obtain equations defined over an entire fixed domain, $\Omega:=\Omega_{\alpha}(t) \cup \Omega_{\beta}(t)$, by prescribing a variable mass density and dynamic viscosity in space and time so that a fluid phase is identified by the value of these quantities on each side of the surface $\mathcal{S}(\mathrm{t})$. Hence

$$
\begin{aligned}
& \rho \partial_{t} \boldsymbol{v}+\rho \operatorname{div}_{\boldsymbol{x}}(\boldsymbol{v} \otimes \boldsymbol{v})=\operatorname{div}_{\boldsymbol{x}} \boldsymbol{T}+\boldsymbol{b}+\boldsymbol{f} \quad \text { in } \quad \Omega, \quad \text { and } \\
& \operatorname{div}_{\boldsymbol{x}} \boldsymbol{v}=0 \quad \text { in } \Omega \text {, }
\end{aligned}
$$

where

$$
\begin{gathered}
\rho(\boldsymbol{x}, t):=\left\{\begin{array}{ll}
\rho_{\alpha} & \forall \boldsymbol{x} \in \Omega_{\alpha}(t), \\
\rho_{\beta} & \forall \boldsymbol{x} \in \Omega_{\beta}(t),
\end{array} \quad \boldsymbol{v}(\boldsymbol{x}, t):= \begin{cases}\boldsymbol{v}_{\alpha} & \forall \boldsymbol{x} \in \mathrm{Clo} \Omega_{\alpha}(t), \\
\boldsymbol{v}_{\beta} & \forall \boldsymbol{x} \in \mathrm{Clo} \Omega_{\beta}(t), \\
\dot{\boldsymbol{x}}=\boldsymbol{v}_{\alpha}=\boldsymbol{v}_{\beta} & \forall \boldsymbol{x} \in \mathcal{S}(t),\end{cases} \right. \\
\boldsymbol{T}(\boldsymbol{x}, t):=\left\{\begin{array}{ll}
\boldsymbol{T}_{\alpha} & \forall \boldsymbol{x} \in \Omega_{\alpha}(t), \\
\boldsymbol{T}_{\beta} & \forall \boldsymbol{x} \in \Omega_{\beta}(t),
\end{array} \quad \boldsymbol{b}(\boldsymbol{x}, t):= \begin{cases}\boldsymbol{b}_{\alpha} & \forall \boldsymbol{x} \in \Omega_{\alpha}(t), \\
\boldsymbol{b}_{\beta} & \forall \boldsymbol{x} \in \Omega_{\beta}(t),\end{cases} \right.
\end{gathered}
$$

and

$$
\boldsymbol{f}(\boldsymbol{x}, t):=\int_{\Omega(t)} \operatorname{div}_{\mathcal{S}}(\sigma \mathbb{I}) \delta\left(\boldsymbol{x}-\boldsymbol{x}_{\mathcal{S}}\right) d s .
$$

The apparent appeal of this reformulation is that the equations of change, specified on $\Omega$, are reduced in number. In addition, it provides a easier way to reason about how to develop approximate solution strategies based on classical partial differential equations defined on a fixed domain. Therefore conventional solution methods based on the partition of fixed domains (meshing) can be designed, in principle. The basic idea is to solve the problem on a fixed domain with an embedded, moving surface $\mathcal{S}(t)$ wherein the source force $\boldsymbol{f}$ is applied. This can be done in two different ways, namely, the interface can be either explicitly tracked or implicitly captured (sec. 3).

The proof that (2.3)-(2.5) is a valid mathematical statement of equivalence to (2.1)-(2.2) hinges in the definition of the continuous velocity field in (2.4), and the source force (2.5). In the latter, the convolution of the interfacial stress divergence with a delta function centered on $\mathcal{S}$, applies a force impulse on the interface which 
reproduces the jump condition (2.2a). Hence, $\boldsymbol{f}(\boldsymbol{x}, t)=\mathbf{0} \forall \boldsymbol{x} \notin \mathcal{S}(t)$. Note that the traction jump in (2.2a), that is, $\llbracket \boldsymbol{T} \cdot \boldsymbol{n} \rrbracket$, is taken into account via the definition of the discontinuous Cauchy stress tensor in (2.4) and (2.3a) and in a weak sense.

In summary, problem (2.3)-(2.5) is an equivalent statement of the discontinuous equations of motion presented in the previous section. However, there exists an obvious difficulty when building any numerical solution scheme based on the above equations, namely, evaluating (2.5) with sufficient accuracy. In practical computations, a variety of ways exist for approximating this integral and for defining $\mathcal{S}$. The particular case wherein a fixed domain is swept by $\mathcal{S}$, produces numerical artifacts called spurious currents which are artificial flow recirculations near the interface due to insufficient accuracy when balancing pressure gradients and interfacial tension (Jamet et al., 2002; Popinet and Zaleski, 1999; Scardovelli and Zaleski, 1999). The adverse effects range from uncontrollable instabilities to difficulties in accurately computing gradients near the interface. In dispersed mixing flows, this can be a significant drawback since it limits the calculation of volume fraction and interfacial area in the long-time regimes.

\subsection{Continuous approximation of the equations of motion}

Most numerical solution methods for (2.3)-(2.5) replace the original discontinuous interface model by a continuous approximation. This entails proposing a particular form of continuous fields for $\rho, \mu, \boldsymbol{b}$, and a volume integral approximate for the source force $\boldsymbol{f}$ area integral. To achieve this objective, the balance equations become

$$
\begin{aligned}
& \rho \partial_{t} \boldsymbol{v}+\rho \operatorname{div}_{\boldsymbol{x}}(\boldsymbol{v} \otimes \boldsymbol{v})=\operatorname{div}_{\boldsymbol{x}} \boldsymbol{T}+\boldsymbol{b}+\boldsymbol{f} \quad \text { in } \quad \Omega, \quad \text { and } \\
& \operatorname{div}_{\boldsymbol{x}} \boldsymbol{v}=0 \quad \text { in } \Omega \text {, }
\end{aligned}
$$

where

$$
\begin{gathered}
\rho(\boldsymbol{x}, t):= \begin{cases}\rho_{\alpha} & \forall \boldsymbol{x} \in \Omega_{\alpha}(t) \mid\left(\boldsymbol{x}-\boldsymbol{x}_{\mathcal{S}}\right) \cdot \boldsymbol{n}>\varepsilon, \\
\rho_{\beta} & \forall \boldsymbol{x} \in \Omega_{\beta}(t) \mid\left(\boldsymbol{x}-\boldsymbol{x}_{\mathcal{S}}\right) \cdot \boldsymbol{n}<\varepsilon, \\
\varphi_{\rho}\left(\rho_{\alpha}, \rho_{\beta}, \boldsymbol{x}\right) & \forall \boldsymbol{x} \in \Omega \mid-\varepsilon \leq\left(\boldsymbol{x}-\boldsymbol{x}_{\mathcal{S}}\right) \cdot \boldsymbol{n} \leq \varepsilon,\end{cases} \\
\mu(\boldsymbol{x}, t):= \begin{cases}\mu_{\alpha} & \forall \boldsymbol{x} \in \Omega_{\alpha}(t) \mid\left(\boldsymbol{x}-\boldsymbol{x}_{\mathcal{S}}\right) \cdot \boldsymbol{n}>\varepsilon, \\
\mu_{\beta} & \forall \boldsymbol{x} \in \Omega_{\beta}(t) \mid\left(\boldsymbol{x}-\boldsymbol{x}_{\mathcal{S}}\right) \cdot \boldsymbol{n}<\varepsilon, \\
\varphi_{\mu}\left(\mu_{\alpha}, \mu_{\beta}, \boldsymbol{x}\right) & \forall \boldsymbol{x} \in \Omega \mid-\varepsilon \leq\left(\boldsymbol{x}-\boldsymbol{x}_{\mathcal{S}}\right) \cdot \boldsymbol{n} \leq \varepsilon,\end{cases}
\end{gathered}
$$




$$
\begin{aligned}
& \boldsymbol{b}(\boldsymbol{x}, t):= \begin{cases}\boldsymbol{b}_{\alpha} & \forall \boldsymbol{x} \in \Omega_{\alpha}(t) \mid\left(\boldsymbol{x}-\boldsymbol{x}_{\mathcal{S}}\right) \cdot \boldsymbol{n}>\varepsilon, \\
\boldsymbol{b}_{\beta} & \forall \boldsymbol{x} \in \Omega_{\beta}(t) \mid\left(\boldsymbol{x}-\boldsymbol{x}_{\mathcal{S}}\right) \cdot \boldsymbol{n}<\varepsilon, \\
\boldsymbol{\varphi}_{\boldsymbol{b}}\left(\boldsymbol{b}_{\alpha}, \boldsymbol{b}_{\beta}, \boldsymbol{x}\right) & \forall \boldsymbol{x} \in \Omega \mid-\varepsilon \leq\left(\boldsymbol{x}-\boldsymbol{x}_{\mathcal{S}}\right) \cdot \boldsymbol{n} \leq \varepsilon .\end{cases} \\
& \boldsymbol{v}(\boldsymbol{x}, t):= \begin{cases}\boldsymbol{v}_{\alpha} & \forall \boldsymbol{x} \in \operatorname{Clo} \Omega_{\alpha}(t), \\
\boldsymbol{v}_{\beta} & \forall \boldsymbol{x} \in \operatorname{Clo} \Omega_{\beta}(t), \\
\dot{\boldsymbol{x}}=\boldsymbol{v}_{\alpha}=\boldsymbol{v}_{\beta} & \forall \boldsymbol{x} \in \mathcal{S}(t),\end{cases}
\end{aligned}
$$

All continuous fields in (2.7), with the exception of the velocity field, interpolate the value of the field in the bulk of the $\beta$ phase to the $\alpha$ phase within a thin region of thickness $\varepsilon>0$. If $\boldsymbol{x}$ is a point near $\mathcal{S}, \boldsymbol{x}_{\mathcal{S}}$ is the point on $\mathcal{S}$ colinear to $\boldsymbol{x}$ along $\boldsymbol{n}\left(\boldsymbol{x}_{\mathcal{S}}\right)$, thus $\left(\boldsymbol{x}-\boldsymbol{x}_{\mathcal{S}}\right) \cdot \boldsymbol{n}=\left\|\boldsymbol{x}-\boldsymbol{x}_{\mathcal{S}}\right\| \forall \boldsymbol{x}$. The interpolants $\varphi_{\rho}, \varphi_{\mu}$, and $\varphi_{\boldsymbol{b}}$ are constructed from an analytical function within the interpolation region around $\mathcal{S}$.

The accuracy of this continuum approximation depends on the preservation of the incompressibility of the fluid and its dynamical viscosity in the region of thickness $\varepsilon$. From mass conservation in the thin region around the interface one obtains

$$
\operatorname{div}_{\boldsymbol{x}} \boldsymbol{v}=-\frac{\mathrm{D}_{\mathrm{t}} \varphi_{\rho}}{\varphi_{\rho}} \quad \forall \boldsymbol{x} \in \Omega \mid-\varepsilon \leq\left(\boldsymbol{x}-\boldsymbol{x}_{\mathcal{S}}\right) \cdot \boldsymbol{n} \leq \varepsilon
$$

where $\mathrm{D}_{\mathrm{t}}(\cdot)$ is the material time derivative. Therefore in view of $(2.6 \mathrm{~b})$ a sufficient condition for maintaining incompressibility in the thin region around the interface is to build the interpolant $\varphi_{\rho}(\cdot)$ such that $\mathrm{D}_{\mathrm{t}} \varphi_{\rho}=0$ on all points in the interpolation region, that is,

$$
\partial_{t} \varphi_{\rho}+\nabla_{\boldsymbol{x}} \varphi_{\rho} \cdot \boldsymbol{v}=0 \quad \forall \boldsymbol{x} \in \Omega \quad \mid-\varepsilon \leq\left(\boldsymbol{x}-\boldsymbol{x}_{\mathcal{S}}\right) \cdot \boldsymbol{n} \leq \varepsilon
$$

for all times.

Note that the flow is isochoric everywhere but it does not imply incompressibility of the fluid in the thin region near the interface unless the interpolant $\varphi_{\rho}$ is compatible through (2.9). An argument similar to (2.9) must hold for the dynamic viscosity field wherein the material derivative must vanish to recover the original constant value. That is, $\varphi_{\mu}$ should be build with the condition that $\mathrm{D}_{\mathrm{t}} \varphi_{\mu}=0$ for all points in the interpolation interfacial region, hence

$$
\partial_{t} \varphi_{\mu}+\nabla_{\boldsymbol{x}} \varphi_{\mu} \cdot \boldsymbol{v}=0 \quad \forall \boldsymbol{x} \in \Omega \mid-\varepsilon \leq\left(\boldsymbol{x}-\boldsymbol{x}_{\mathcal{S}}\right) \cdot \boldsymbol{n} \leq \varepsilon .
$$

The interpolant for the body force $\varphi_{\boldsymbol{b}}$ can be obtained from $\varphi_{\rho}$ for the typical gravitational force.

The interpolants $\varphi_{\rho}$ and $\varphi_{\mu}$ are called compatible if they satisfy (2.9) and (2.10) for all times. 
The continuous approximation (2.6)-(2.7) induces a segregated solution approach for evolving the explicit representation of the interface $\mathcal{S}\left(t_{0}\right)$ given at a particular instant in time, $t_{0}$. The equations are solved for $\boldsymbol{v}\left(\boldsymbol{x}, t_{0}\right)$ and $p\left(\boldsymbol{x}, t_{0}\right)$ using a previous value for the mass density, dynamic viscosity, body force, interfacial force, and velocity fields; in addition to boundary conditions. Once the new velocity and pressure fields are computed, the interface is updated according to

$$
d_{t} \boldsymbol{x}_{\mathcal{S}}=\boldsymbol{v}\left(\boldsymbol{x}_{\mathcal{S}}, t_{0}\right) \quad \forall \boldsymbol{x}_{\mathcal{S}} \in \mathcal{S}\left(t_{0}\right)
$$

The new locus of $\mathcal{S}\left(t_{1}\right)$ produces the fields $\rho\left(\boldsymbol{x}, t_{1}\right), \mu\left(\boldsymbol{x}, t_{1}\right), \boldsymbol{b}\left(\boldsymbol{x}, t_{1}\right)$, and $\boldsymbol{f}\left(\boldsymbol{x}, t_{1}\right)$ by virtue of (2.7) and (2.5) which can be used, in the next iteration, to solve for the new velocity and pressure fields at the new time instant $t_{1}$.

There are various ways to proceed with the interface update (2.11) which requires extensive geometric operations. The next sections elaborate on existing approaches and the current method we are developing.

\section{Approximate solution methods}

This section elaborates on some details of the numerical algorithms used in our method to solve the approximation described in Section 2.2. As stated earlier, the approach uses an explicit time evolution of the interface sweeping the domain occupied by the fluid phases. The approach consists of a loop over two main tasks:

- Calculation of the velocity and pressure fields in a fixed domain with given continuous mass density, viscosity fields, and interfacial momentum source.

- Update of the position of the interface with the newly computed interfacial velocity field, and update of the mass density and dynamic viscosity fields.

The first task entails the standard calculation of the solution of the Navier-Stokes equations with variable mass density, dynamic viscosity, interfacial force, and body force fields. These fields only vary appreciably near the interface. However accounting of this variation is critical for the correct evolution of the interface. The description of this first step is the subject of Section 3.1

The second task consists of an explicit time-step for advancing the position of the interface. This is a complex task when using a front-tracking method to represent and evolve a mathematical surface. This task must accommodate various algorithms for reconstructing the interface as it evolves and changes its topology. The description of this task is presented in the remaining subsections. 
Zhou, Ray, Lim, Wang, de Almeida, Glimm, Li, and Jiao

\subsection{Solution of the equations of motions on a fixed domain}

Various numerical methods can be used effectively to solve the continuous equations (2.6)-(2.7) for fixed $\Omega_{\alpha}\left(t_{0}\right)$ and $\Omega_{\beta}\left(t_{0}\right)$. If initial values for the fields $\rho\left(\boldsymbol{x}, t_{0}\right), \mu\left(\boldsymbol{x}, t_{0}\right)$, $\boldsymbol{f}\left(\boldsymbol{x}, t_{0}\right)$, and $\boldsymbol{b}\left(\boldsymbol{x}, t_{0}\right)$ are given, and initial and boundary conditions for $\boldsymbol{v}\left(\boldsymbol{x}, t_{0}\right)$ applied, an approximate solution can be computed for $\boldsymbol{v}\left(\boldsymbol{x}, t_{1}\right)$ and $p\left(\boldsymbol{x}, t_{1}\right)$ by solving (2.6) on the fixed phase domains.

In this work we applied a projection method with a fractional step using an intermediate velocity. Since the pioneering work of Chorin $(1968,1969)$, many high-order projection methods were derived by Bell et al. $(1989,1991)$, Kim and Moin (1985), and Kan (1986). All projection methods have three steps: First, one obtains an intermediate velocity field $\boldsymbol{v}^{*}$ by solving a convection-diffusion equation with proper boundary conditions. Then a (Hodge) projection is performed to enforce the divergence-free condition (incompressibility) for the velocity field. This step is implemented by solving an elliptic equation. Lastly, the pressure is updated via a Poisson equation (Brown et al., 2001). Additional information is provided later in this report in Section 3.4.

\subsection{Numerical description of fluid interfaces}

Many methods have been proposed to represent and evolve an interface between two immiscible fluids. Of these, the volume of fluid (VOF), the level set, and fronttracking methods are the most popular. A complete review is beyond the scope of this report. Readers are referred to the papers of Hirt and Nichols (1981), Sethian (1996), Glimm et al. (1998a, 2000), Tryggvason et al. (2001), Scardovelli and Zaleski (1999). When compared to the front capturing methods (VOF and level set), front tracking preserves a more accurate interface representation with less numerical dissipation. In addition, the implicit methods suffer from the drawback that they are unable to capture interface details near or below the resolution of the underlying grid. For those physics problems such as fluid mixing which needs an accurate representation of the interface, front tracking is a better choice in our opinion. A comparison of these three methods (Du et al., 2006) concluded that the front tracking to be more accurate and faster to converge for a number of benchmark problems.

We use front tracking methods to simulate multiphase flows, based on the front tracking code FronTier. This is a robust library for the geometrical manipulation of a mathematical surface coupled with multiple partial differential equations. It has been employed in a variety of multiphase simulations (Du et al., 2006; Glimm et al., 1998b, 2000) for problems dominated by a geometrically complex dynamic interface. For example, extensive simulation studies of the Rayleigh-Taylor instability (Lim et al., 2010; Liu et al., 2006, 2007) delivered agreement with experiments in the overall growth rate as defined by the mixing growth parameter. 
The Fron Tier package provides algorithms for geometric construction and reconstruction of a surface embedded in the surrounding three-dimensional space. Surface normal, curvature, area, enclosed volume, and statistical information of disconnected surfaces are calculated robustly with higher order accuracy. These geometric operations are necessary for enabling the evolution of fluid interfaces in practical simulations.

Topological bifurcations of an interface occur frequently in many applications and require special front tracking methods. Our FronTier package uses a triangular representation of a surface. That is, a three-dimensional triangular surface mesh is used. Triangles on the interface must be allowed to reconnect with each other after crossing when dynamically convected by the surrounding fluid flow. Three methods are implemented in FronTier to handle topological transitions. The grid free method (GF) (Glimm et al., 1998b) is accurate but is prone to logical errors. A grid based method (GB) (Glimm et al., 2000) is robust but suffers from excessive interpolation and smoothing errors. The most advanced, the locally grid-based (LGB) method (Du et al., 2006), combines the advantages of the GF and GB methods. It identifies degenerated triangles on the propagated surface, isolates them, and preserves the intersections of the surface with the grid cell edges to allow for a GB reconstruction locally near the defective region. Triangles neighboring this region are removed giving rise to a gap between the pristine interface and the reconstructed part. The major geometry task is to re-seal this gap.

We recently (Bo et al., 2011) improved the LGB reconstruction. The essential feature of the improvement is to reduce the size of the GB region, which previously was the smallest rectangular solid containing a tangled set; and in the case of overlaps, the smallest rectangular solid containing the overlap, etc. In order to keep the triangular mesh topologically valid, we impose two constraints on the triangular mesh: (1) an edge of a triangle connects with at most one triangle; (2) an non-boundary vertex has only one associated list of triangles formed by linking successively adjacent triangles.

To parallelize the improved LGB algorithm we construct ghost cells, typically a few layers thick, so that after ghost cell communications, each processor utilizes only local information to reconstruct the interface contained within its local cells. If the span of the tangling is too large for the ghost cells, we collect the cells containing the tangled region onto one processor for resolution. Because such situations occur very rarely, this fall back strategy does not affect the efficiency.

\subsection{Brief survey of methods for fluid flows with interfaces}

In recent decades, a large effort has been devoted to interface-resolving methods for the solution of the multiphase, incompressible Navier-Stokes equations. The Im-

mersed Boundary Method (IB), Immersed Interface Method (IIM), Embedded Bound- 
Zhou, Ray, Lim, Wang, de Almeida, Glimm, Li, and Jiao

ary Method (EBM) and the Ghost Fluid Method (GFM) are the most popular methods for solving this class of fluid flow problems.

The IB method, introduced by Peskin (1977) to model blood flow in a human heart, uses a discrete delta function to spread the singular force on the interface to a Cartesian grid by means of a numerical width multiple of the grid spacing. This approach was extended (Unverdi and Tryggvason, 1992) to treat three-dimensional, multi-phase incompressible fluid flows including complex topological changes. Sussman et al. (1994) replaced the front tracking description of the interface (Unverdi and Tryggvason, 1992) with a level set description (see review articles in Mittal and Iaccarino, 2005; Peskin, 2002).

The IB method has been successfully applied to many fluid and biological problems. It is first-order due to the smoothing of the interface. The numerical solution is continuous at the interface even if the actual interface conditions imply that the solution should be discontinuous.

The IIM, introduced by LeVeque and Li (1994), uses a regular Cartesian grid with an interface represented by marker points. Instead of using a discrete delta functions and/or Heaviside functions, the velocity and pressure jump conditions at the interface are preserved and coupled into the finite difference scheme resulting in a high-order numerical method. This method was initially implemented in one-dimensional and two-dimensional elliptic equations with discontinuous coefficients and singular source terms. The IIM was then generalized to many types of equations with jump conditions at the interface, and a review of this body of work is found elsewhere ( $\mathrm{Li}$ and Ito, 2006; Li, 2003).

Recently (Tan et al., 2008, 2009) the IIM was generalized to apply to the incompressible Navier-Stokes equation with discontinuous viscosity, using the jump conditions of Ito and Li (2006); Lai and Li (2001). However, the method does not allow discontinuities in the density for the Navier-Stokes solutions. It is second-order accurate in the absence of density discontinuities. With density discontinuities, which must be smeared over some length scale, as in the IB method, the method is first-order accurate. The IIM relies on a local coordinate system which is quite complicated and whose generalization to three or more fluid phases appears to be difficult.

The GFM is a sharp interface method which preserves the jump conditions and uses them to construct ghost fluid states for updating each phase independently. As with the IB method, it is a first-order method but it differs from the IB in its treatment of the interface. It was used for a Poisson's equation (Liu et al., 2000) and for a multi-phase incompressible flow (Kang et al., 2000).

The GFM could be used to treat three-dimensional, multi-phase incompressible fluid flow, including the effect of viscosity, surface tension and gravity, eliminating the numerical smearing prevalent in the discrete delta function formulation of the IBM. The GFM is relatively simple to implement. The sharp interface allows complete 
physics including a discontinuous density. Unlike the IIM, it involves linear systems with symmetric matrices of coefficients which can be solved by many fast solvers. The GFM is only first-order accurate because of a simplification used in the jump conditions when constructing the ghost states (details in Fedkiw et al., 1999; Terashima and Tryggvason, 2009; Tryggvason et al., 2001).

The EBM uses a Cartesian grid as the computational domain with the irregular boundary embedded in the grid. The states are calculated at grid cell centers even if they are outside of the boundary. It is proved both theoretically and numerically that this method is second-order accurate. The EBM was first introduced for a Poisson's equation on an irregular domain in Johansen and Colella (1998). It was generalized to parabolic equations with a moving boundary (McCorquodale et al., 2001), three-dimensional elliptic and parabolic equations (Schwartz et al., 2006), hyperbolic conservation laws (Colella et al., 2006), and compressible (Kupiainen and Sjogreen, 2009) and incompressible (Barad et al., 2009) single-phase, Navier-Stokes equations on irregular domains.

The EBM was extended to a two-sided interface, as opposed to a one-sided boundary (Wang et al., 2010), with application to two-phase flows. The EBM incorporates the jump conditions for elliptic and parabolic equations with additional states defined not only at the cell center, but also at the interface patch center, to yield a second-order accurate algorithm for both the interface problem and for the irregular boundary problem in a unified framework. It is also easily generalized to three or more phases through additional jump conditions and states. The difficulty in obtaining second-order accuracy, especially in the case of a large density discontinuity at the interface, is associated with the projection step.

\subsection{Front tracking with the immersed boundary method}

We use the front tracking method referred in Section 3.2 combined with the IB method referred in Section 3.3, similar to Unverdi and Tryggvason (1992). With the interface represented by a triangular mesh, the interpolant (2.7) for mass density and viscosity is built with the aid of a Heaviside function (Liu et al., 2000)

$$
H(\phi)= \begin{cases}0, & \phi<-\varepsilon, \\ \frac{1}{2}+\frac{\phi}{2 \varepsilon}+\frac{1}{2 \pi} \sin \left(\frac{\pi \phi}{\varepsilon}\right), & -\varepsilon \leq \phi \leq \varepsilon, \\ 1, & \phi>\varepsilon\end{cases}
$$


where $\phi:=\left(\boldsymbol{x}-\boldsymbol{x}_{\mathcal{S}}\right) \cdot \boldsymbol{n}_{\overrightarrow{\beta \alpha}}$ is the distance to the interface in the direction of the surface normal. We compute the density and viscosity close to the interface as

$$
\begin{aligned}
& \rho(\phi)=\rho_{\beta}+\left(\rho_{\alpha}-\rho_{\beta}\right) H(\phi), \\
& \mu(\phi)=\mu_{\beta}+\left(\mu_{\alpha}-\mu_{\beta}\right) H(\phi) .
\end{aligned}
$$

For approximating the evaluation of the volume interfacial force (2.5) we use a discrete delta function formulation defined in (Kim and Peskin, 2007). That is, for each grid cell in $\Omega, I_{i j k}$, the interfacial force per unit of volume given by the IB method at the cell center equals to

$$
\boldsymbol{F}_{i j k}=\sum_{l} D\left(\boldsymbol{x}_{i j k}-\boldsymbol{x}^{(l)}\right) \boldsymbol{f}^{(l)}
$$

where $\boldsymbol{x}_{i j k}$ and $\boldsymbol{x}^{(l)}$ are the position of the grid cell center and the centroid of the front triangular element with index $l$, respectively. The discrete delta function in (2.5) is approximated by $D(\cdot)$, above, and

$$
\boldsymbol{f}^{(l)}:=2 \mathcal{H}^{(l)} \sigma \boldsymbol{n}^{(l)} \Delta s^{(l)}
$$

is the interfacial force on the $l$ th interface triangle, $\mathcal{H}^{(l)}$ is the mean curvature of the interface at the centroid of the corresponding triangle, $\boldsymbol{n}^{(l)}$ is the unit normal of the triangle, and $\Delta s^{(l)}$ is the surface area of the associated triangle.

Here the mean curvature for each triangle $\mathcal{H}^{(l)}$ is the average of the mean curvatures on the three vertices which are calculated by a polynomial fitting given by Jiao and Zha (2008) with a second-order accuracy. In the cylindrical coordinate system, used for our tests and simulations, a coordinate transformation on the points positions was made as an input to the algorithm (Jiao and Zha, 2008) that calculates the mean curvature in Cartesian coordinates. As the transformation for point position is exact, the order of accuracy for computing the mean curvature in cylindrical coordinates was preserved.

Specificaly, in a rectangular Cartesian coordinate system, for each surface point $\boldsymbol{x}_{0}$ on $\mathcal{S}$, a local coordinate system $u, v, w$ is established based on the approximated unit normal $\boldsymbol{n}\left(\boldsymbol{x}_{0}\right)$ vector. The local coordinate system prompts the orthogonal transformation matrix

$$
\boldsymbol{Q}:=\left[\begin{array}{lll}
\boldsymbol{t}_{1} & \boldsymbol{t}_{2} & \boldsymbol{m}
\end{array}\right]
$$

where $\boldsymbol{t}_{1}$ and $\boldsymbol{t}_{2}$ are the unit vectors, in the global coordinate system $x, y, z$, along the positive direction of the $u$ and $v$ axes while $\boldsymbol{m}:=\boldsymbol{t}_{1} \times \boldsymbol{t}_{2}$ is the unit vector along the positive $w$ direction. In the local coordinate system, a set of points $\left\{\left(u_{i}, v_{i}, W_{i}\right)\right\}$ 
around $\boldsymbol{x}_{0}$ are generated via a polynomial interpolation from a Taylor series expansion with degree of fitting $d$ as

$$
W \approx \sum_{p=0}^{d} \sum_{j, k \geq 0}^{j+k=p} c_{j k} \frac{u^{j} v^{k}}{j ! k !} .
$$

The coefficients $c_{j k}$ are solved for by evaluating $W$ at the points $W_{i}:=W\left(u_{i}, v_{i}\right)$ and creating a weighted least squares problem. A robust QR factorization with safeguard is used to solve the problem and from the gradient and Hessian of $W$,

$$
\nabla W:=\left(\begin{array}{l}
W_{u} \\
W_{v}
\end{array}\right), \quad \boldsymbol{H}:=\left(\begin{array}{ll}
W_{u u} & W_{u v} \\
W_{v u} & W_{v v}
\end{array}\right),
$$

respectively, the local unit normal $\hat{\boldsymbol{n}}$ and mean curvature $\mathcal{H}$ can be computed. The normal unit vector in the global retangular Cartesian coordinate system is computed by the transformation $\boldsymbol{n}=\boldsymbol{Q} \cdot \hat{\boldsymbol{n}}$ while the mean curvature $\mathcal{H}$ is an invariant of the curvature tensor therefore independent of the coordinate system.

For the cylindrical coordinate system used in this work, a set of points $\left\{\left(r_{i}, \theta_{i}, z_{i}\right)\right\}$ is selected around the point $\boldsymbol{x}_{0}:=\left(r_{0}, \theta_{0}, z_{0}\right)$, and transformed into the $x, y, z$ coordinate system

$$
x_{i}=r_{i} \cos \theta_{i}, \quad y_{i}=r_{i} \sin \theta_{i}, \quad z_{i}=z_{i},
$$

which are used as the input points for the polynomial fitting of $W$. Hence by transforming the normal vector $\left(n_{x}, n_{y}, n_{z}\right)$, obtained from a high-order interpolation of $\mathrm{W}$, in cylindrical coordinates $\left(n_{r}, n_{\theta}, n_{z}\right)$ via

$$
\begin{aligned}
& n_{\theta}=-n_{x} \sin \theta_{0}+n_{y} \cos \theta_{0}, \\
& n_{r}=n_{x} \cos \theta_{0}+n_{y} \sin \theta_{0}, \text { and } \\
& n_{z}=n_{z},
\end{aligned}
$$

the accuracy of the surface normal and mean curvature calculation is preserved.

In this work the particular discrete delta function used to compute the volumetric interfacial force (3.4) has the following form

$$
D\left(\boldsymbol{x}_{i j k}-\boldsymbol{x}^{(l)}\right)=\frac{\delta_{h}\left(\frac{\left(_{1}^{i j k}-x_{1}^{(l)}\right.}{h_{1}}\right) \delta_{h}\left(\frac{x_{2}^{i j k}-x_{2}^{(l)}}{h_{2}}\right) \delta_{h}\left(\frac{x_{3}^{i j k}-x_{3}^{(l)}}{h_{3}}\right)}{V\left(I_{i j k}\right)}
$$

where $V\left(I_{i j k}\right)$ is the volume of cell $I_{i j k}$ and the function $\delta_{h}$ is given by (Kim and Peskin, 2007):

$$
\delta_{h}(r)=\left\{\begin{array}{lll}
\frac{3-2|r|+\sqrt{1+4|r|-4 r^{2}}}{8}, & \text { if } & |r|<1, \\
\frac{5-2|r|-\sqrt{-7+12|r|-4 r^{2}}}{8}, & \text { if } & 1 \leqslant|r|<2, \\
0, & \text { if } & 2 \leqslant|r| .
\end{array}\right.
$$


Zhou, Ray, Lim, Wang, de Almeida, Glimm, Li, and Jiao

When implementing formula (3.4), we select a triangle on the interface, choose the cells within a radius of three cell diameters around the triangle, and distribute the interfacial force onto the cells using the discrete delta function (3.7). In this way the interfacial force is approximated by a volume integral

$$
\int_{\Omega} 2 \mathcal{H} \sigma \boldsymbol{n} \delta\left(\boldsymbol{x}-\boldsymbol{x}_{\mathcal{S}}\right) d v=\int_{\mathcal{S}} 2 \mathcal{H} \sigma \boldsymbol{n} d s=\int_{\Omega} \boldsymbol{F}_{i j k} d v .
$$

The Courant-Friedrichs-Lewy (CFL) condition related to interfacial tension from Brackbill et al. (1992) is implemented as a requirement of stability

$$
\Delta t_{1}<\left[\frac{\langle\rho\rangle(\Delta \ell)^{3}}{2 \pi \sigma}\right]^{1 / 2},
$$

where $\langle\rho\rangle$ is the average density, $\Delta \ell$ is the smallest cell edge and $\sigma$ is the interfacial tension coefficient. Together with the convective time step restriction

$$
\Delta t_{2} \leq \frac{1}{\frac{\left.u u\right|_{\max }}{\Delta x}+\frac{|v|_{\max }}{\Delta y}+\frac{|w|_{\max }}{\Delta z}}
$$

where $|u|_{\max },|v|_{\max }$ and $|w|_{\max }$ are the maximum Cartesian velocity components magnitude, the final time step follows

$$
\Delta t=C_{\mathrm{CFL}} \times \min \left\{\Delta t_{1}, \Delta t_{2}\right\},
$$

where $C_{\mathrm{CFL}}$ is the CFL number (less than 1).

For solving the incompressible Navier-Stokes equations with a variable density and viscosity given by the IB method, we follow the projection method advanced in Bell et al. $(1989,1991)$, which is second-order accurate in velocity for single-phase incompressible fluid flow and known as PMI (Brown et al., 2001). This method is only first-order accurate in pressure, however the alternate method used here, PMII, is second-order accurate in pressure provided appropriate boundary conditions are used.

We use a second-order Godunov scheme to solve the nonlinear advection term. This algorithm generates a source term in the diffusion equation. These two steps are solved in a coupled manner to achieve higher order time accuracy. The implicit second-order time integration Crank-Nicholson scheme is used to solve the diffusion equation. The Laplacian equation in the projection step is solved by a standard fivepoint discretization. Both the linear systems formed in the diffusion and projection step are sparse matrices, solved in parallel using PETSc (Balay et al., 2008).

We use the GMRES linear solver for the diffusion step and the BiCGSL linear solver for the projection step to obtain a faster convergence rate. However, the 
BiCGSL algorithm is not theoretically stable and occasionally it does not converge, in which case we use GMRES instead after a large residual has been observed for the iterative linear solver.

A comment about the calculation of the Cauchy stress tensor is in order. The continuous approximation of the governing equations of motion (2.6)-(2.7) calls for a continuously varying dynamical viscosity. This requires the deviatoric part of the stress tensor to be computed as $\mu(\boldsymbol{x}, t)\left(\nabla_{\boldsymbol{x}} \boldsymbol{v}+\nabla_{\boldsymbol{x}} \boldsymbol{v}^{T}\right)$. Therefore this term is differentiated by the divergence operator in the momentum balance equations.

With the velocity field computed from the incompressible Navier-Stokes equations (2.6)-(2.7), we obtain the velocity of the interface points by interpolation from velocity data given at regular grid points. As we propagate the interface, the new positions of the interface points are calculated by solving the ODE (2.11) using the forward Euler method.

\section{Verification of one-phase flow by the method of manufactured solutions}

In this section we consider the order of accuracy of the projection method (Sec. 3.1) for solving the incompressible Navier-Stokes equations. As mentioned in Section 3.4, the method tested here uses PMII which is a second-order projection method for singlephase, incompressible fluid flow. We use initial and boundary data and associated source terms for which the exact solution is known. This method is applied in 2D and 3D Cartesian coordinates and in 3D cylindrical coordinates. The exact solution and data for the 2D solution we use here were employed previously (Brown et al., 2001). The 3D exact solution comes from Ethier and Steinman (1994) and a modification is needed for the case of 3D cylindrical coordinates.

\subsection{Time-dependent solutions in 2D Cartesian coordinates}

With the domain $\Omega=[0,1] \times[0,1]$ and parameters $\rho=1$ and $\mu=1$, the Navier-Stokes equations are augmented with a forcing term so that the functions

$$
\begin{aligned}
u= & \cos (2 \pi(x-\omega(t)))\left(3 y^{2}-2 y\right) \\
v= & 2 \pi \sin (2 \pi(x-\omega(t))) y^{2}(y-1) \\
p= & -\frac{\omega^{\prime}(t)}{2 \pi} \sin (2 \pi(x-\omega(t)))(\sin (2 \pi y)-2 \pi y+\pi) \\
& -\cos (2 \pi(x-\omega(t)))(-2 \sin (2 \pi y)+2 \pi y)-\pi),
\end{aligned}
$$


with $\omega(t)=1+\sin \left(2 \pi t^{2}\right)$, become an exact solution (Brown et al., 2001). The boundary condition for the $x$ direction is periodic and Dirichlet for the $y$ direction.

The required source terms are derived by substituting (4.1)-(4.3) into (A.35)(A.37). The formulas for the source terms, derived using Maple with $\mathrm{C}$ code generated directly from symbolic computation, are too long to be inserted here. A uniform time step of $\Delta t=0.4 h$ was used, corresponding to a CFL number of 0.4 . The errors are calculated in both $L_{1}$ and $L_{\infty}$ norms at time 0.5 for $N \times N$ grids with $N$ equal to 10 , 20, 40 and 80. The errors and order of accuracy for the $u$ and $v$ component of the velocity field and the pressure $p$ are shown in Tables 4.1-4.3. The results are very close to the theoretically predicted second-order accuracy.

Table 4.1: Verification of convergence rate of the $u$-component of the velocity for 2D NSE in Cartesian coordinates.

\begin{tabular}{c|c|c|c|c} 
& $L_{1}$ error & Order in $L_{1}$ norm & $L_{\infty}$ error & Order in $L_{\infty}$ norm \\
\hline $10 \times 10$ & 0.02505001 & NA & 0.05749996 & NA \\
\hline $20 \times 20$ & 0.00596152 & 2.0711 & 0.02271585 & 1.3399 \\
\hline $40 \times 40$ & 0.00138930 & 2.1013 & 0.00620064 & 1.8732 \\
\hline $80 \times 80$ & 0.00033850 & 2.0371 & 0.00159809 & 1.9561
\end{tabular}

Table 4.2: Verification of convergence rate of the $v$-component of the velocity for 2D NSE in Cartesian coordinates.

\begin{tabular}{c|c|c|c|c} 
& $L_{1}$ error & Order in $L_{1}$ norm & $L_{\infty}$ error & Order in $L_{\infty}$ norm \\
\hline $10 \times 10$ & 0.02187048 & NA & 0.04523046 & NA \\
\hline $20 \times 20$ & 0.00706664 & 1.8890 & 0.01307569 & 1.6299 \\
\hline $40 \times 40$ & 0.00179580 & 1.9671 & 0.00334820 & 1.9764 \\
\hline $80 \times 80$ & 0.00045103 & 1.9879 & 0.00084932 & 1.9933
\end{tabular}

\subsection{Time-dependent solutions in 3D Cartesian coordinates}

We set the domain $\Omega=[-1,1] \times[-1,1] \times[-1,1]$, and choose parameters $\rho=1$ and $\mu=1$. The Navier-Stokes equations are augmented with a forcing term in order to 
Table 4.3: Verification of convergence rate of the pressure $p$ for 2D NSE in Cartesian coordinates.

\begin{tabular}{c|c|c|c|c} 
& $L_{1}$ error & Order in $L_{1}$ norm & $L_{\infty}$ error & Order in $L_{\infty}$ norm \\
\hline $10 \times 10$ & 0.20987610 & NA & 0.91315993 & NA \\
\hline $20 \times 20$ & 0.04786609 & 2.1325 & 0.29326793 & 1.6391 \\
\hline $40 \times 40$ & 0.00970739 & 2.3018 & 0.06626968 & 2.1458 \\
\hline $80 \times 80$ & 0.00219919 & 2.1421 & 0.01504217 & 2.1393
\end{tabular}

the solution be (Ethier and Steinman, 1994):

$$
\begin{aligned}
u= & -a\left[e^{a x} \sin (a y+d z)+e^{a z} \cos (a x+d y)\right] e^{-d^{2} t} \\
v= & -a\left[e^{a y} \sin (a z+d x)+e^{a x} \cos (a y+d z)\right] e^{-d^{2} t} \\
w= & -a\left[e^{a z} \sin (a x+d y)+e^{a y} \cos (a z+d x)\right] e^{-d^{2} t} \\
p= & -\frac{a^{2}}{2}\left[e^{2 a x}+e^{2 a y}+e^{2 a z}+2 \sin (a x+d y) \cos (a z+d x) e^{a(y+z)}\right. \\
& +2 \sin (a y+d z) \cos (a x+d y) e^{a(z+x)} \\
& \left.+2 \sin (a z+d x) \cos (a y+d z) e^{a(x+y)}\right] e^{-2 d^{2} t} .
\end{aligned}
$$

We set $a=1$ and $d=1$, with Dirichlet boundary conditions on all six boundaries.

As in the 2D test, we derive the source term using Maple from (A.38)-(A.41). The maximum flow speed of the solution is about 10 , so a uniform time step of $\Delta t=0.05 h$ is used, corresponding to a CFL number of 0.5 to make the algorithm stable. The errors are calculated in both $L_{1}$ and $L_{\infty}$ norms at time 0.2 for $N \times N \times N$ grids with $N$ equal to 10, 20, 40 and 80. In Table 4.4, we omit the $v$ and $w$ velocity components as their convergence rates are similar to that of the $u$ velocity component.

Table 4.4: Verification of convergence rate of $u$-component velocity for 3D NSE in Cartesian coordinates.

\begin{tabular}{c|c|c|c|c} 
& $L_{1}$ error & Order in $L_{1}$ norm & $L_{\infty}$ error & Order in $L_{\infty}$ norm \\
\hline $10 \times 10$ & 0.04540364 & NA & 0.01354348 & NA \\
\hline $20 \times 20$ & 0.01177651 & 1.9469 & 0.00373252 & 1.8594 \\
\hline $40 \times 40$ & 0.00299105 & 1.9772 & 0.00127001 & 1.5553 \\
\hline $80 \times 80$ & 0.00075277 & 1.9904 & 0.00038637 & 1.7168
\end{tabular}


Table 4.5: Verification of convergence rate of pressure $p$ for 3D NSE in Cartesian coordinates.

\begin{tabular}{c|c|c|c|c} 
& $L_{1}$ error & Order in $L_{1}$ norm & $L_{\infty}$ error & Order in $L_{\infty}$ norm \\
\hline $10 \times 10$ & 0.56668074 & NA & 0.57665525 & NA \\
\hline $20 \times 20$ & 0.14954512 & 1.9220 & 0.53674946 & 0.1035 \\
\hline $40 \times 40$ & 0.03779002 & 1.9845 & 0.34788539 & 0.6256 \\
\hline $80 \times 80$ & 0.00947893 & 1.9952 & 0.19797179 & 0.8133
\end{tabular}

We observe in Table 4.5 that the $L_{\infty}$ norm error of pressure $p$ is only first-order accurate when using Dirichlet boundary condition for all boundaries. The normal mode analysis of Brown et al. (2001) for second-order accuracy in pressure requires the boundary conditions to be periodic except in one direction. Since the 3-D exact solution we used does not satisfy this requirement, the obtained reduced order of accuracy in the $L_{\infty}$ norm appears to be justified; this is not a verified assertion.

\subsection{Time-dependent solutions in $3 \mathrm{D}$ cylindrical coordinates}

To generalize the second-order projection method (Bell et al., 1989, 1991) to cylindrical coordinates, we treat the terms which are multiplied by $\rho$ as the convection terms and those multiplied by $\mu$ as diffusion terms. First, we use a second-order Gudonov-type scheme to discretize the advection term; the result becomes a source term when solving the diffusion equation using the Crank-Nicholson scheme. For the projection step, an ordinary central difference discretization is used for the Poisson equation with a pure Neumann boundary condition. Finally, we update the pressure and the velocity to obtain a second-order accurate velocity field and a first-order accurate pressure when non-periodic boundary conditions are used.

The manufactured solution in cylindrical coordinate is

$$
\begin{aligned}
& u_{\theta}(r, \theta, z)=\frac{1}{12}\left(-r^{2} \sin \theta+r^{2} \cos \theta-z^{2} \sin \theta+z^{2} \cos \theta\right) e^{-t} \\
& u_{z}(r, \theta, z)=-\frac{1}{6} z r(\cos \theta+\sin \theta) e^{-t} \\
& u_{r}(r, \theta, z)=-\frac{1}{12}\left(r^{2} \cos \theta+r^{2} \sin \theta+z^{2} \cos \theta+z^{2} \sin \theta\right) e^{-t} \\
& p(r, \theta, z)=\frac{1}{12}\left(r^{2}+z^{2}\right) e^{-t} .
\end{aligned}
$$

We set the computational domain to be $\theta \in[0,2 \pi], z \in[-1,1], r \in[1,2]$ and the other parameters as in the $3 \mathrm{D}$ manufactured solution in Cartesian coordinates. The 
source term was derived by substituting the exact solution into the incompressible Navier-Stokes equations in cylindrical coordinates (A.42)-(A.45). The results, summarized in Tables 4.6-4.9, are very close to the theoretically predicted convergence rates.

Table 4.6: Verification of convergence rate of $u_{\theta}$ for 3D NSE in cylindrical coordinates.

\begin{tabular}{c|c|c|c|c} 
& $L_{1}$ error & Order in $L_{1}$ norm & $L_{\infty}$ error & Order in $L_{\infty}$ norm \\
\hline $10 \times 10$ & 0.21461366 & NA & 0.03974838 & NA \\
\hline $20 \times 20$ & 0.05242220 & 2.0335 & 0.01018509 & 1.9643 \\
\hline $40 \times 40$ & 0.01297461 & 2.0145 & 0.00256724 & 1.9882 \\
\hline $80 \times 80$ & 0.00323912 & 2.0020 & 0.00064171 & 2.0002
\end{tabular}

Table 4.7: Verification of convergence rate of $u_{z}$ for 3D NSE in cylindrical coordinates.

\begin{tabular}{c|c|c|c|c} 
& $L_{1}$ error & Order in $L_{1}$ norm & $L_{\infty}$ error & Order in $L_{\infty}$ norm \\
\hline $10 \times 10$ & 0.01729539 & NA & 0.00352590 & NA \\
\hline $20 \times 20$ & 0.00483825 & 1.8378 & 0.00135969 & 1.3747 \\
\hline $40 \times 40$ & 0.00123408 & 1.9710 & 0.00035601 & 1.9333 \\
\hline $80 \times 80$ & 0.00031296 & 2.0020 & 0.00011209 & 1.6673
\end{tabular}

Again we see here that the convergence rate of $L_{\infty}$ norm error for pressure is first-order accurate rather than second-order when we refine the mesh because of the Dirichlet boundary condition on all six boundaries; not a verified assertion.

\section{Verification and validation of one-phase annular Couette flow}

The Taylor-Couette flow regime occurs in the annulus between differentially rotating concentric cylinders, most often with the inner cylinder rotating and the outer cylinder 
Table 4.8: Verification of convergence rate of $u_{r}$ for 3D NSE in cylindrical coordinates.

\begin{tabular}{c|c|c|c|c} 
& $L_{1}$ error & Order in $L_{1}$ norm & $L_{\infty}$ error & Order in $L_{\infty}$ norm \\
\hline $10 \times 10$ & 0.01569499 & NA & 0.00346419 & NA \\
\hline $20 \times 20$ & 0.00427474 & 1.8764 & 0.00144748 & 1.2590 \\
\hline $40 \times 40$ & 0.00109416 & 1.9660 & 0.00053794 & 1.4280 \\
\hline $80 \times 80$ & 0.00028311 & 1.9504 & 0.00017048 & 1.6553
\end{tabular}

Table 4.9: Verification of convergence rate of $p$ for 3D NSE in cylindrical coordinates.

\begin{tabular}{c|c|c|c|c} 
& $L_{1}$ error & Order in $L_{1}$ norm & $L_{\infty}$ error & Order in $L_{\infty}$ norm \\
\hline $10 \times 10$ & 7.41387743 & NA & 0.76635043 & NA \\
\hline $20 \times 20$ & 1.78645195 & 2.0531 & 0.19345287 & 1.9860 \\
\hline $40 \times 40$ & 0.43545378 & 2.0365 & 0.05218918 & 1.8902 \\
\hline $80 \times 80$ & 0.10759368 & 2.0169 & 0.02173881 & 1.2535
\end{tabular}

fixed. The flow becomes unstable when the rotation rate exceeds a critical value (fig. 5.1) (Lueptow, 2009) and a secondary motion in the form of vortices appears in the cross section of the primary flow. The critical rotation rate is different depending on whether the inner or the outer cylinder is moving. Since the pioneering work of Taylor (1923), in which linear stability analysis was used to predict the appearance of Taylor vortices, the Taylor-Couette flow has been studied extensively, theoretically, experimentally and computationally.

Taylor-Couette flow is important not only because of its applications, such as the design of a viscometer, fluid mixture apparatus, and in biology; it also has been studied to observe flow patterns during the transition from laminar to turbulent flow. With increasing Reynolds number, the flow undergoes a series of transitions from circular Couette flow, to axially periodic Taylor vortex flow, to a state with timedependent waves imposed on the vortices (wavy Taylor vortex flow) and to chaotic and turbulent Taylor vortex flow. A visualization of Taylor vortex flow is displayed in Figure 5.1 (Lueptow, 2009).

Two-phase Couette flow also has multiple applications, including liquid-gas oxygenators, and, as considered here, liquid-liquid extraction. However in various practical liquid-liquid systems of industrial interest the mass density and dynamic viscosity 

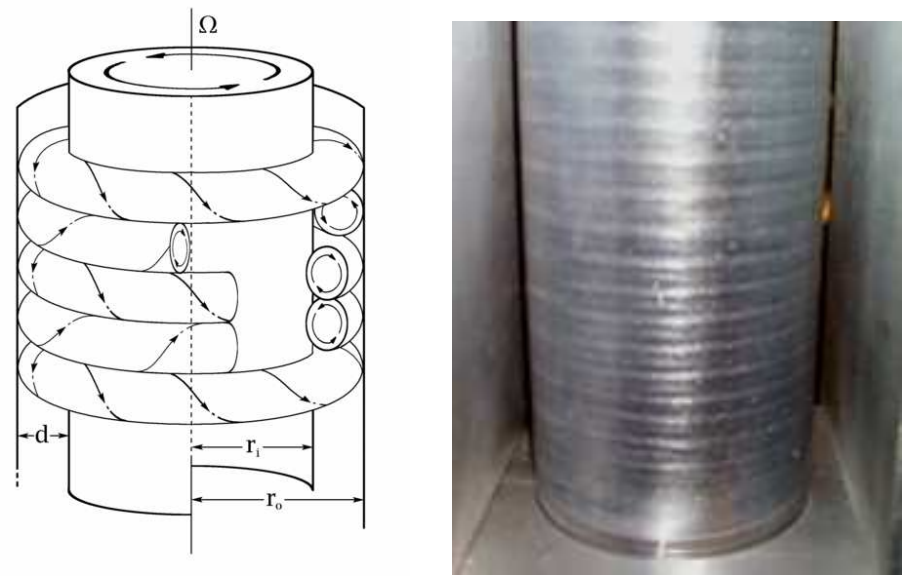

Fig. 5.1: Left: Schematic diagram of counter-rotating axisymmetric vortices of Taylor-Couette flow (@2000, Mike Minbiole and Richard M. Lueptow). Right: Axisymmetric Taylor vortices visualized using titanium dioxide-coated mica flakes (C)2002, Alp Akonur and Richard M. Lueptow)

differences combined with fast cylinder rotation produce an extremely complex flow pattern of mixing that has received far less attention than the one-phase Taylor vortex flow counterpart; we return to this topic in Section 7.

Section 5.1 develops the analytic solution for the annular Couette flow used for code verification. Section 5.2 presents simulation results for the transition from circular Couette flow regime to the Taylor-Couette flow regime. The critical value for this transition is predicted from 3-D simulations as demonstrated in Section 5.3, while fully turbulent flow is studied in Section 5.4.

The dimensionless parameters which characterize the system are: the radii ratio $\eta=R_{\mathrm{i}} / R_{\mathrm{o}}$ of the inner to the outer cylinders respectively; the cylinder height to the gap width aspect ratio $\Gamma:=\frac{L_{z}}{R_{\mathrm{o}}-R_{\mathrm{i}}}$, where the gap width between cylinders is also denoted $d:=R_{\mathrm{o}}-R_{\mathrm{i}}$; the Reynolds number $R e$; and the Taylor number $T$. The definitions for the last two parameters vary in the literature, here we use

$$
\begin{aligned}
R e & :=\frac{\Omega_{\mathrm{i}} R_{\mathrm{i}}\left(R_{\mathrm{o}}-R_{\mathrm{i}}\right)}{\nu}, \text { and } \\
T & :=4 R e^{2}\left(\frac{1-\eta}{1+\eta}\right),
\end{aligned}
$$

where $\Omega_{\mathrm{i}}$ is the rotation speed of the inner cylinder and $\nu$ is the kinematic viscosity of the fluid. 


\subsection{Steady-state 1-D Couette flow in an annular sector}

For a sub-critical Reynolds number, Couette flow is laminar. Under this assumption, the Navier-Stokes equations simplify to one-dimensional flow. In cylindrical coordinates, we derived an exact solution for an infinite cylinder $(\Gamma=\infty)$,

$$
\begin{aligned}
& v_{\theta}=C_{1} r+\frac{C_{2}}{r} \\
& v_{z}=0 ; \\
& v_{r}=0 ; \\
& P=\rho\left[\frac{C_{1}^{2}\left(r^{2}-R_{\mathrm{i}}^{2}\right)}{2}+2 C_{1} C_{2} \ln \frac{r}{R_{\mathrm{i}}}-\frac{C_{2}^{2}}{2}\left(\frac{1}{r^{2}}-\frac{1}{R_{\mathrm{i}}^{2}}\right)\right]+P_{0},
\end{aligned}
$$

with

$$
\begin{aligned}
C_{1} & =\frac{\Omega_{\mathrm{i}} R_{\mathrm{i}}^{2}}{R_{\mathrm{i}}^{2}-R_{\mathrm{o}}^{2}} \\
C_{2} & =-C_{1} R_{\mathrm{o}}^{2} \\
P_{0} & =P\left(R_{\mathrm{i}}\right) .
\end{aligned}
$$

This solution was used as a verification and convergence rate test for the numerical solution method of the three-dimensional Navier-Stokes equations. For this purpose, we used periodic boundary conditions in the $z$ and $\theta$ directions and no slip conditions on the boundaries in the $r$ direction. We set the computational domain to be $\theta \in$ $[0,0.628] \mathrm{rad}, z \in[0,0.628] \mathrm{cm}, r \in[2.538,3.166] \mathrm{cm}$, and $\Omega_{\mathrm{i}}=0.05 \mathrm{rad} / \mathrm{s}(0.48$ RPM). A time-dependent, three-dimensional simulation was performed using $\rho=$ $1.0 \mathrm{~g} / \mathrm{cm}^{3}$ and $\nu=0.0089 \mathrm{~cm}^{2} / \mathrm{s}$ which resulted in $R e=9.0$. The initial velocity and pressure fields for the simulation were enforced using the foregoing exact solution fields. The simulation results obtained for a small $R e$ compared well with the exact solution (5.3)-(5.6) obtained for $R e=0$. Errors were calculated in both $L_{1}$ and $L_{\infty}$ norms at time $1 \mathrm{~s}$ (0.008 rotations) for $N \times N \times N$ grids with $N$ equal to 10, 20, 40 and 80 . As $u_{z}=0$ within round off error, we omit analysis of this variable and display convergence results for $u_{\theta}, u_{r}$ and the pressure $p$ in Tables 5.1-5.3. All convergence results confirm a second-order accurate solution algorithm.

\subsection{Transition from 1-D to 3-D Taylor vortices}

A qualitative demonstration of the existence of the vortex transition is made in this section at the Reynolds number $R e=2686$. The simulation parameters were $R_{\mathrm{i}}=$ $2.538 \mathrm{~cm}, R_{\mathrm{o}}=3.166 \mathrm{~cm}, \eta=R_{\mathrm{i}} / R_{\mathrm{o}}=0.8, \Omega_{\mathrm{i}}=15 \mathrm{rad} / \mathrm{s}($ or $38.07 \mathrm{~cm} / \mathrm{s}$, or 
Table 5.1: Verification of the convergence rate of $u_{\theta}$ for $1 \mathrm{D}$ Couette flow.

\begin{tabular}{c|c|c|c|c} 
& $L_{1}$ error & Order in $L_{1}$ norm & $L_{\infty}$ error & Order in $L_{\infty}$ norm \\
\hline $10 \times 10$ & $8.9689427 \mathrm{e}-06$ & NA & $4.2889586 \mathrm{e}-05$ & NA \\
\hline $20 \times 20$ & $2.3392513 \mathrm{e}-06$ & 1.9445 & $1.2213023 \mathrm{e}-05$ & 1.8122 \\
\hline $40 \times 40$ & $5.8993089 \mathrm{e}-07$ & 1.9874 & $3.2289907 \mathrm{e}-06$ & 1.9193 \\
\hline $80 \times 80$ & $1.4774364 \mathrm{e}-07$ & 1.9974 & $8.2839859 \mathrm{e}-07$ & 1.9627
\end{tabular}

Table 5.2: Verification of the convergence rate of $u_{r}$ for $1 \mathrm{D}$ Couette flow.

\begin{tabular}{c|c|c|c|c} 
& $L_{1}$ error & Order in $L_{1}$ norm & $L_{\infty}$ error & Order in $L_{\infty}$ norm \\
\hline $10 \times 10$ & $3.2098794 \mathrm{e}-06$ & NA & $1.3955543 \mathrm{e}-05$ & NA \\
\hline $20 \times 20$ & $2.3019156 \mathrm{e}-07$ & 3.8016 & $3.2426953 \mathrm{e}-06$ & 2.1056 \\
\hline $40 \times 40$ & $1.4560125 \mathrm{e}-08$ & 3.9827 & $4.0839172 \mathrm{e}-07$ & 2.9892 \\
\hline $80 \times 80$ & $9.1318711 \mathrm{e}-10$ & 3.9950 & $5.0681518 \mathrm{e}-08$ & 3.0104
\end{tabular}

Table 5.3: Verification of the convergence rate of $p$ for 1D Couette flow.

\begin{tabular}{c|c|c|c|c} 
& $L_{1}$ error & Order in $L_{1}$ norm & $L_{\infty}$ error & Order in $L_{\infty}$ norm \\
\hline $10 \times 10$ & $4.0876435 \mathrm{e}-06$ & NA & $2.2784380 \mathrm{e}-05$ & NA \\
\hline $20 \times 20$ & $7.9287009 \mathrm{e}-07$ & 2.3661 & $4.6255377 \mathrm{e}-06$ & 2.3004 \\
\hline $40 \times 40$ & $1.9027897 \mathrm{e}-07$ & 2.0590 & $1.2303281 \mathrm{e}-06$ & 1.9106 \\
\hline $80 \times 80$ & $4.6547912 \mathrm{e}-08$ & 2.0313 & $3.2019695 \mathrm{e}-07$ & 1.9420
\end{tabular}


143.2 RPM), $\rho=1.0 \mathrm{~g} / \mathrm{cm}^{3}, \nu=0.0089 \mathrm{~cm}^{2} / \mathrm{s}$ and

$$
R e=\frac{\Omega_{\mathrm{i}} R_{\mathrm{i}}\left(R_{\mathrm{o}}-R_{\mathrm{i}}\right)}{\nu}=2686.3, \quad T=4 R e^{2}\left(\frac{1-\eta}{1+\eta}\right)=3.2 \times 10^{6} .
$$

The critical Reynolds number for the transition to vortex flow is 94.7 (Prima and Swinney, 1985), so this simulation is well above the transition point. The computational domain is $\theta \in[0,0.628] \mathrm{rad}, z \in[0,1.256] \mathrm{cm}, r \in[2.538,3.166] \mathrm{cm}$. We used periodic boundary conditions for both the $\theta$ and $z$ directions and a no slip boundary condition for the velocity in the $r$ direction. The initialization of the velocity and pressure fields were as in (5.3)-(5.6), that is, the unperturbed laminar solution for one-phase Couette flow. A simulation up to $t=2 s$ was performed (about 5 rotations of the inner cylinder). The grid size for this simulation is $40 \times 80 \times 40$. Streamlines of the $v_{r}$ and $v_{z}$ velocity component fields indicate the formation of the Taylor vortices (fig. 5.2) which is consistent with the linear stability theory.

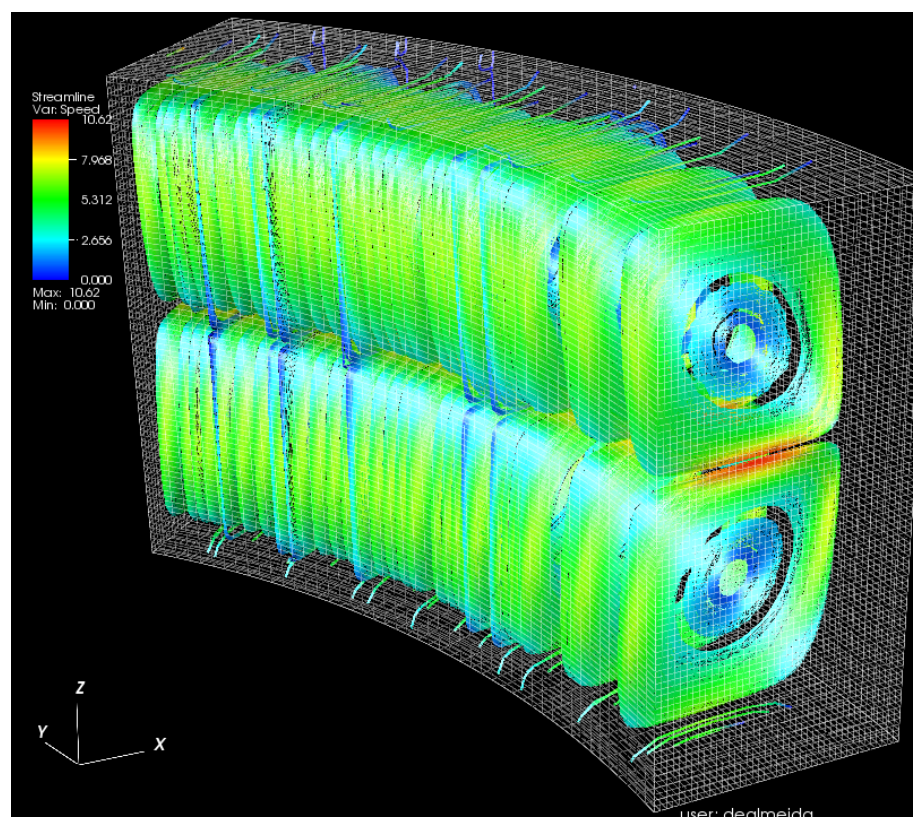

Fig. 5.2: Computed velocity streamlines in the $r-z$ plane of a Taylor vortex flow at $R e=2686.3$ (i.e. $\left.T=3.2 \times 10^{6}\right)$.

\subsection{Verifying the Couette flow perturbation growth rate}

The axisymmetric linear stability analysis of 3-D small perturbations (Sec. A.2) imposed on a 1-D Couette base flow predicts the minimum Reynolds number for the 
growth of the disturbances for any wave number. The analysis also predicts the growth rate to be time dependent. Therefore in this section we present a verification of the growth of the disturbances (by means of numerical analysis) through a direct time-dependent, 3-D simulation of the evolution of the initial 1-D Couette flow field.

Using (5.3)-(5.6) as the initial conditions for a 3-D flow simulation in the same annular sector of the previous section (Sec. 5.2), we calculate the growth rate

$$
\begin{aligned}
K(t) & :=\frac{\sqrt{\left\langle v_{r}\right\rangle^{2}+\left\langle v_{z}\right\rangle^{2}}}{\left|\left\langle v_{\theta}\right\rangle\right|}, \\
& \approx \frac{\sqrt{\left(u^{\prime}\right)^{2}+\left(w^{\prime}\right)^{2}}}{\left|V+v^{\prime}\right|}, \\
& \approx\left[e^{\beta t} \frac{e^{i \lambda z} \sqrt{u^{2}(r)+w^{2}(r)}}{\left|V+v^{\prime}\right|}\right], \\
\ln K(t) & \approx \beta t+C(r, \theta, z) .
\end{aligned}
$$

where $\mathrm{C}$ is a constant, in time, according to the linear stability analysis assumption (A.21). Thus the slope $\beta$ of the graph of $\ln K$ versus $t$ gives the growth rate of the fundamental mode for each Reynolds number (figs. 5.3 and 5.4).

As indicated in figures 5.3 and $5.4, K$ has two flat sections and one linearly growing section; the slope is calculated in the interval $10^{-6}<K<10^{-4}$. The linearly growing section is associated to an exponential growth of the instability $e^{\beta t}$ from linear stability analysis. The first flat section results from the initialization of the instability, which is seeded by numerical grid effects, and not by a perturbation at the maximally growing wave length. The results confirm that at a higher Reynolds number the flow disturbances grow faster. The second flat section corresponds to the approach to the steady state flow with Taylor vortices.

Figure 5.5 shows the growth rate $\beta$ as a function of Reynolds number. The growth rate for supercritical disturbances grows with the Reynolds number Re. As $\beta \rightarrow 0$ then $R e \rightarrow R_{c}$ and by virtue of a first order Taylor expansion we know from linear stability analysis that $\beta(R e)=c_{0}\left(R e-R_{c}\right)$ when $R e-R_{c}$ is small. Thus from a linear fitting to the slope of $\beta(R e)$ we compute $c_{0}$ and find $R_{c}$ setting $\beta=0$ (inset of fig. 5.5). We obtain the value $R_{c}=95.35$, which is very close to the theoretical value of $R_{c}=94.7$ (Prima and Swinney, 1985).

\subsection{Turbulent Taylor-Couette flow: verification and valida- tion}

In this section a comparison of flow simulation with and without turbulence modeling is made against experimental results and direct numerical simulation of the Navier- 


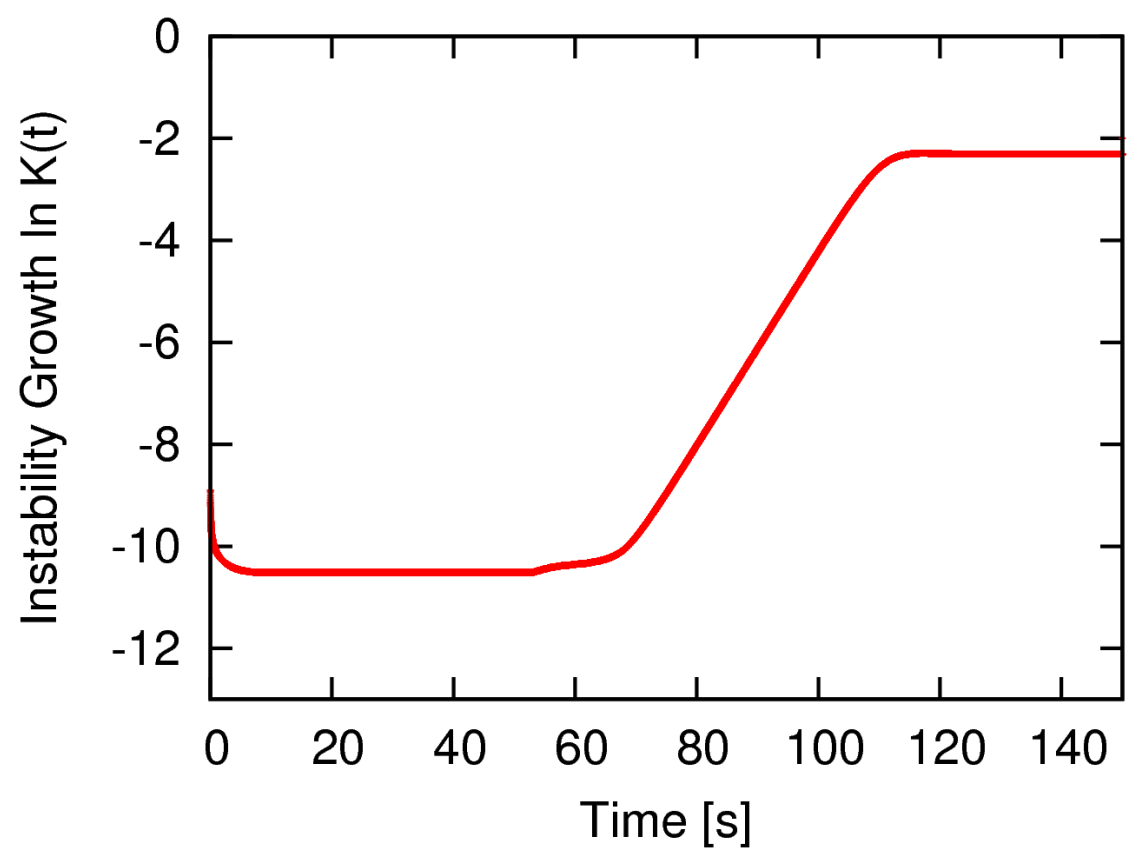

Fig. 5.3: Computed instability growth $\ln K$ at $R e=125$ for a Taylor-Couette flow.

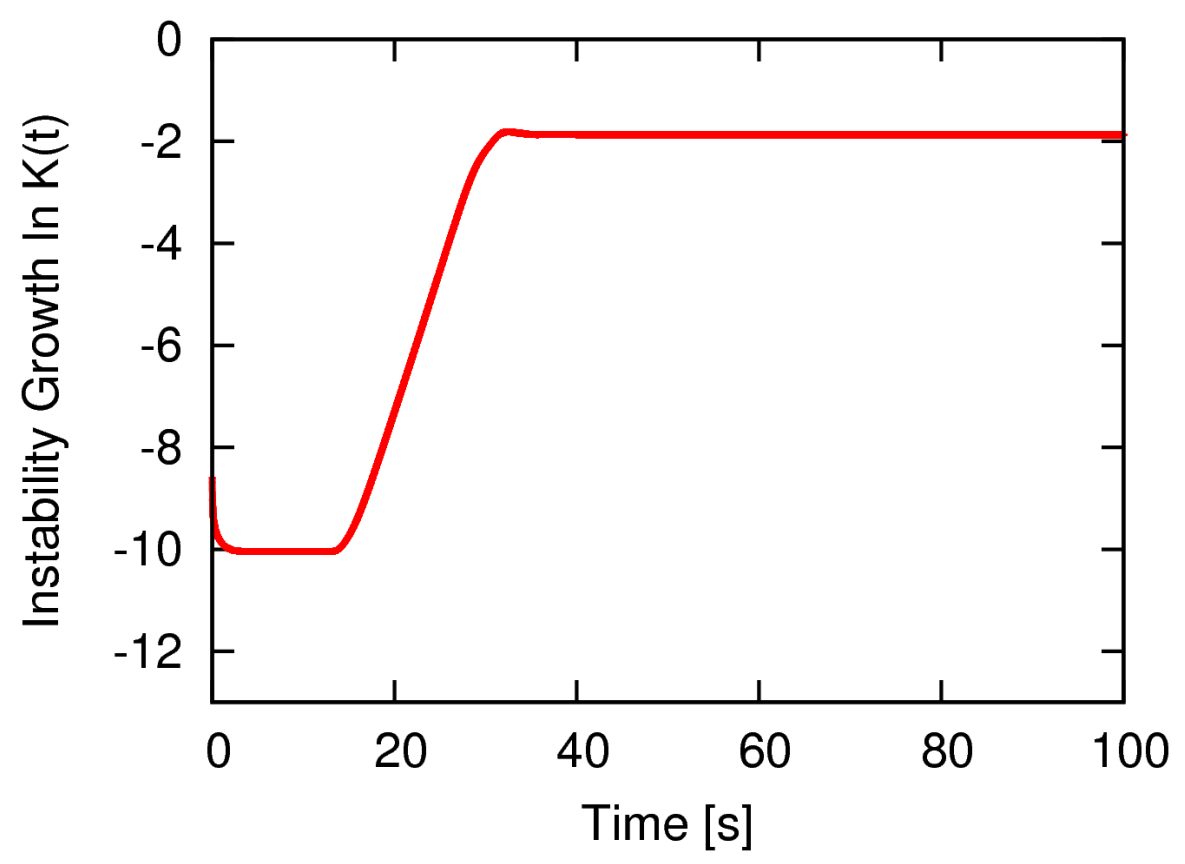

Fig. 5.4: Computed instability growth $\ln K$ at $R e=200$ for a Taylor-Couette flow. 


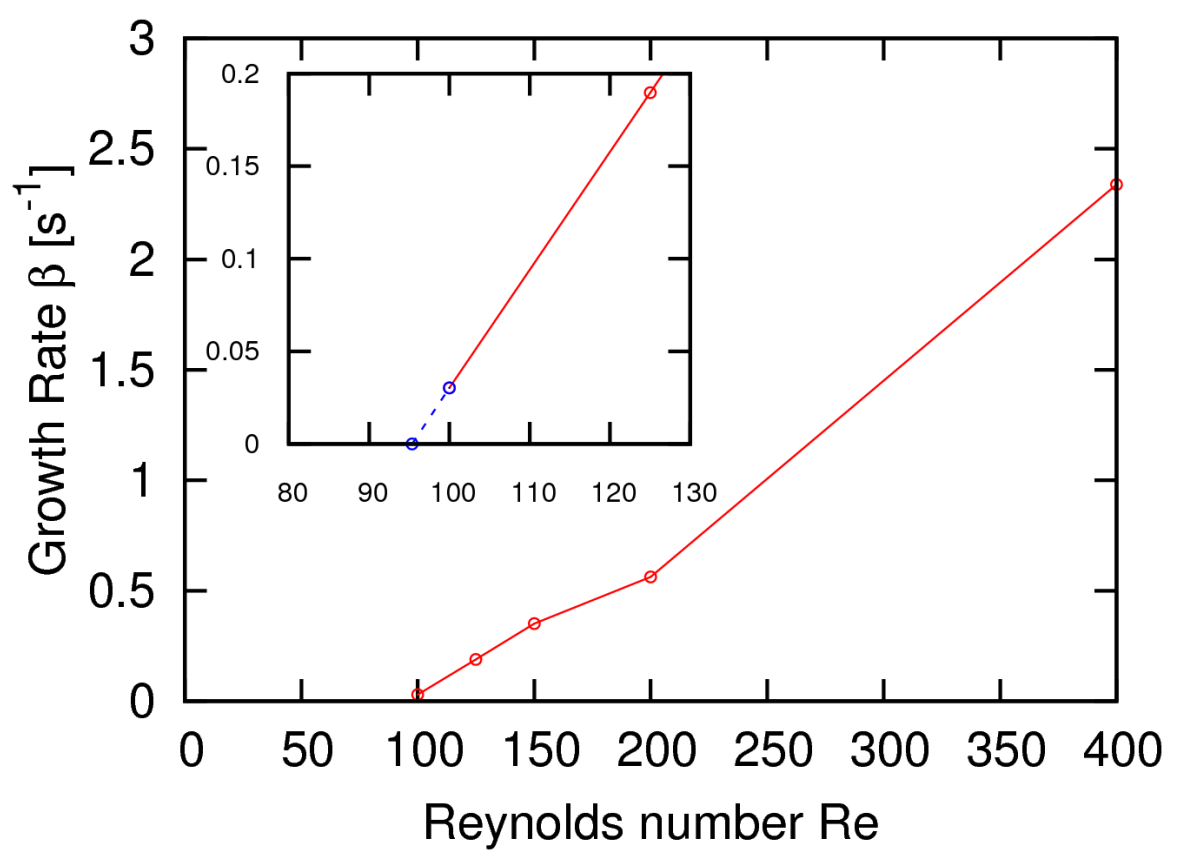

Fig. 5.5: Calculated growth rate vs. Reynolds number for a Taylor-Couette flow. Critical Reynolds number $R_{c}=95.35$ found by extrapolation.

Stokes equations for the Taylor-Couette flow at $R e=8000$. The same geometry as in the previous Section 5.3 was used. Our results are compared against the experimental results of Smith and Townsend (1983), and the direct numerical simulation (DNS) results of Dong (2007). The governing equations of the turbulence modeling (large eddy simulation, LES) subgrid scale model used are presented in the Appendix A.4.

The dimensionless values of the parameters in our simulation are $L_{z}=\pi, R_{\mathrm{i}}=1$, $R_{\mathrm{o}}=2$, density $\rho=1$ and dynamic viscosity $\mu=1 / 8000$. The Reynolds number in this case is $R e=U_{1}\left(R_{\mathrm{o}}-R_{\mathrm{i}}\right) \rho / \mu=8000$. Periodic boundary conditions are imposed in the axial and azimuthal direction with no-slip boundary conditions at the inner and outer cylinder. The initialization is a uniform velocity field $U=0.5$. The simulation was not terminated until a statistically-stationary state was achieved and at this stage, data was collected for a period of 250 dimensionless time units. We used three sets of meshes $64 \times 32 \times 32,64 \times 64 \times 32$ and $128 \times 64 \times 32$, in the coordinate directions: $\theta, z, r$.

Figure 5.6 compares the mean angular momentum $\left\langle u_{\theta} r\right\rangle / U_{1} R_{\mathrm{i}}$ with experimental values $(R e=8698)$ which are available only for the region near the inner cylinder. The most refined mesh agrees with the experimental data for the region near the inner cylinder (Smith and Townsend, 1983). Figure 5.6 also shows the agreement of our results with DNS (Dong, 2007) as the mesh is refined. 
Zhou, Ray, Lim, Wang, De Almeida, Glimm, Li, and Jiao

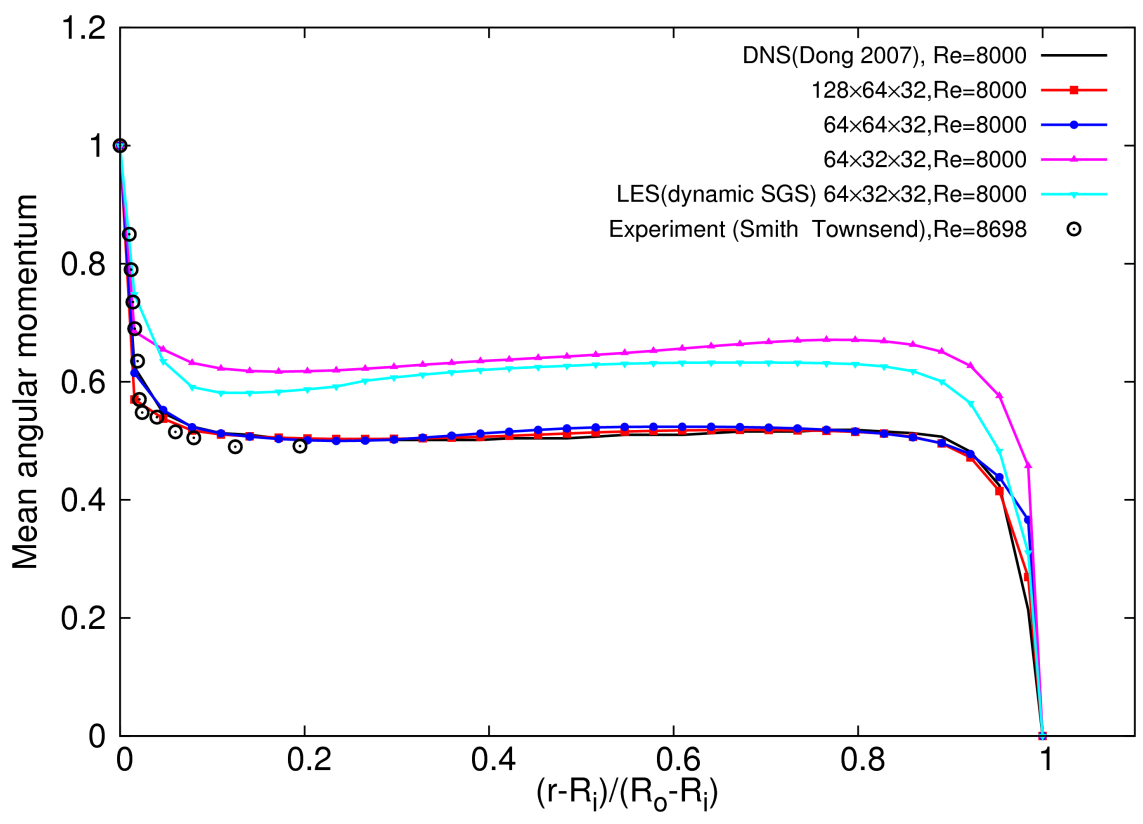

Fig. 5.6: Mean angular momentum $\left\langle v_{\theta} r\right\rangle / U_{1} R_{\mathrm{i}}$ for a turbulent (LES model) singlephase Couette flow.

Similarly, in Figure 5.7, the profile of the mean azimuthal velocity $\left\langle u_{\theta}\right\rangle / U_{1}$ agrees with that of the DNS simulation upon mesh refinement. The results using the subgrid scale model show slightly improved convergence. Results in both figures compare well with DNS (Dong, 2007) and with the experimental result of Smith and Townsend (1983). This verification and validation test evaluates positively the implementation of our fluid flow solver.

\section{Verification of two-phase annular Couette flow}

Two-phase Couette flow also undergoes a transition from 1-D circular flow to 3-D toroidal flow wherein secondary vortices appear in both phases. If the Reynolds number is large enough, as in practical applications of mixing, the flow regime is dramatically different than one-phase vortex flow. For the particular case of two immiscible liquids, vortices disappear and an intricate interfacial shape evolves (Sec. 7).

When the flow is circular 1-D in the presence of gravity pointing in the axial direction, the incompressible Navier-Stokes equations in cylindrical coordinates simplify to a one, non-zero velocity component in the azimuthal direction. In addition, if the pressure gradient is only a function of the radial direction, an exact solution can be 


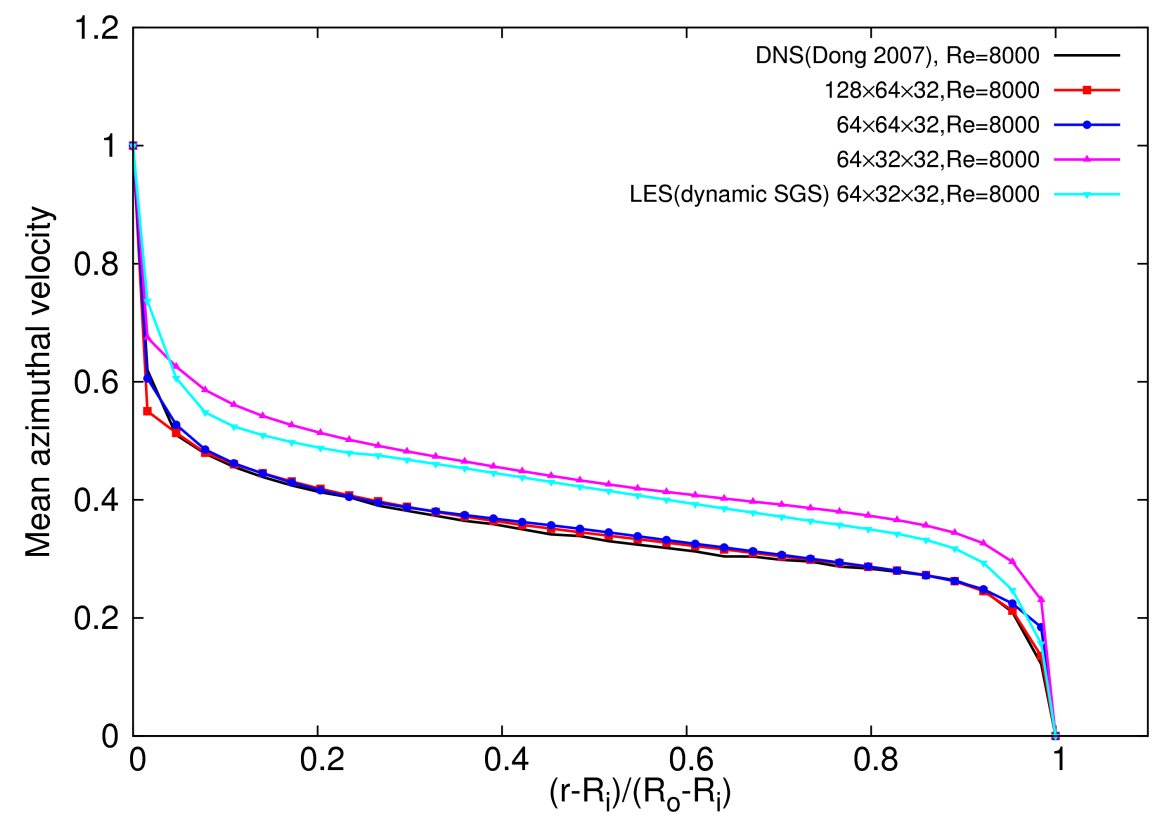

Fig. 5.7: Mean azimuthal velocity $\left\langle v_{\theta}\right\rangle / U_{1}$ for a turbulent (LES model) single-phase Couette flow.

constructed for the velocity and pressure unknowns as function of the radius and axial coordinates.

Denoting $\mu_{1}$ and $\mu_{2}$ the kinematic viscosity of fluid 1 and fluid 2, respectively, and $R_{\mathrm{I}}$ the radial position of the interface, the following constants of integration are obtained

$$
\begin{aligned}
& B_{1}=\frac{-\Omega_{\mathrm{i}} R_{\mathrm{I}}^{2}}{1-R_{\mathrm{Ii}}^{2}+\mu_{12} R_{\mathrm{Io}}^{2}-\mu_{12}} \\
& A_{1}=\Omega_{\mathrm{i}}-\frac{B_{1}}{R_{\mathrm{i}}^{2}}, \\
& B_{2}=\mu_{12} B_{1},
\end{aligned}
$$

and

$$
A_{2}=-\mu_{12} \frac{B_{1}}{R_{\mathrm{o}}^{2}} .
$$

where $\mu_{12}=\mu_{1} / \mu_{2}, R_{\mathrm{Ii}}=R_{\mathrm{I}} / R_{\mathrm{i}}, R_{\mathrm{Io}}=R_{\mathrm{I}} / R_{\mathrm{o}}$. Then in the fluid phase $1, r \leq R_{\mathrm{I}}$, 
the azimuthal velocity and pressure fields are

$$
\begin{aligned}
v_{\theta, 1}(r)= & A_{1} r+\frac{B_{1}}{r} \\
P_{1}(r, z)= & \rho_{1}\left[\frac{A_{1}^{2}}{2}\left(r^{2}-R_{\mathrm{i}}^{2}\right)+2 A_{1} B_{1} \ln \frac{r}{R_{\mathrm{i}}}-\frac{B_{1}^{2}}{2}\left(\frac{1}{r^{2}}-\frac{1}{R_{\mathrm{i}}^{2}}\right)\right] \\
& -\rho_{1} g\left(z-z_{0}\right)+P_{0},
\end{aligned}
$$

and in the fluid phase $2, r \geq R_{\mathrm{I}}$,

$$
\begin{aligned}
v_{\theta, 2}(r)= & A_{2} r+\frac{B_{2}}{r} \\
P_{2}(r, z)= & \rho_{1}\left[\frac{A_{1}^{2}}{2}\left(R_{\mathrm{I}}^{2}-R_{\mathrm{i}}^{2}\right)+2 A_{1} B_{1} \ln \frac{R_{\mathrm{I}}}{R_{\mathrm{i}}}-\frac{B_{1}^{2}}{2}\left(\frac{1}{R_{\mathrm{I}}^{2}}-\frac{1}{R_{\mathrm{i}}^{2}}\right)\right] \\
& +\rho_{2}\left[\frac{A_{2}^{2}}{2}\left(r^{2}-R_{\mathrm{I}}^{2}\right)+2 A_{2} B_{2} \ln \frac{r}{R_{\mathrm{I}}}-\frac{B_{2}^{2}}{2}\left(\frac{1}{r^{2}}-\frac{1}{R_{\mathrm{I}}^{2}}\right)\right] \\
& -\rho_{1} g\left(z-z_{0}\right)-\frac{\sigma}{R_{\mathrm{I}}}+P_{0} .
\end{aligned}
$$

where

$$
C_{1}=\frac{\Omega_{\mathrm{i}} R_{\mathrm{i}}^{2}}{R_{\mathrm{i}}^{2}-R_{\mathrm{o}}^{2}}, \quad C_{2}=-C_{1} R_{\mathrm{o}}^{2} .
$$

The pressure jump across the interface is computed by subtracting (6.8) from (6.6)

$$
P_{1}\left(R_{\mathrm{I}}, z\right)-P_{2}\left(R_{\mathrm{I}}, z\right)=\frac{\sigma}{R_{\mathrm{I}}}
$$

The above expressions are used as a verification and convergence rate test for our front tracking solution method as described next. Periodic boundary conditions in the $z$ and $\theta$ directions are enforced in conjunction with no slip boundary conditions on the $r$-direction boundaries. The computational annular sector domain is $\theta \in[0,0.628] \mathrm{rad}$, $z \in[0,0.628] \mathrm{cm}$ and $r \in[2.538,3.166] \mathrm{cm}$, and the following values were selected

$$
\begin{array}{llrl}
\rho_{1} & =0.811 \mathrm{~g} / \mathrm{cm}^{3}, & \rho_{2}=1.03 \mathrm{~g} / \mathrm{cm}^{3}, \\
\mu_{1}=57.6 \mathrm{~g} / \mathrm{cm} \mathrm{s}(5760 \mathrm{cP}), & \mu_{2}=36.72 \mathrm{~g} / \mathrm{cm} \mathrm{s}(3672 \mathrm{cP}) .
\end{array}
$$

with the angular speed of the inner cylinder is $\Omega_{\mathrm{i}}=0.22367 \mathrm{rad} / \mathrm{s}(2.1249 \mathrm{RPM})$ which results in the Reynolds number $R e=0.01$ to approximate a zero inertial flow. The gravity $g$ is neglected and the interface position at $r=2.9 \mathrm{~cm}$ is used as the initial condition.

In addition, three different values of interfacial tension coefficients were used in various tests, namely, $\sigma=0, \sigma=0.001$, and $\sigma=10 \mathrm{dyn} / \mathrm{cm}$. Relative errors of 
the interface position, velocity field $u_{\theta}$, and pressure field $p$ were calculated using reference values in both $L_{1}$ and $L_{\infty}$ norms for $N \times N \times N$ grids with $N$ equal to 10,20 and 40. In the calculation of relative errors, the exact position of the interface was used, i.e., $R_{\mathrm{I}}=2.9 \mathrm{~cm}$, the speed of the inner cylinder was chosen as the velocity scale, $\Omega_{\mathrm{i}} R_{\mathrm{i}}=0.56768 \mathrm{~cm} / \mathrm{s}$, and for the reference pressure, the maximum pressure difference $\Delta p$ in the computation domain was used; this value is different for each of the three test cases. The CFL number was adjusted (reduced) for this two-phase flow test case.

\subsection{Couette flow with no interfacial tension $(\sigma=0)$}

Without interfacial tension the pressure is continuous. The errors are computed at time $t=28.1 \mathrm{~s}$ ( 1 revolution) when the reference pressure is $\Delta p=0.023257 \mathrm{dyn} / \mathrm{cm}^{2}$. Results for 3D simulations are collected in tables 6.1, 6.2, and 6.3.

Table 6.1: Solution verification and calculation of the convergence rate of the interface position for $1 \mathrm{D}$, two-phase Couette flow without interfacial tension, $\sigma=0$.

\begin{tabular}{c|c|c|c|c} 
& $L_{1}$ error & Order in $L_{1}$ norm & $L_{\infty}$ error & Order in $L_{\infty}$ norm \\
\hline $10 \times 10$ & $2.6857 \mathrm{e}-8$ & NA & $2.6866 \mathrm{e}-8$ & NA \\
\hline $20 \times 20$ & $3.1387 \mathrm{e}-9$ & 3.0971 & $3.2010 \mathrm{e}-9$ & 3.0692 \\
\hline $40 \times 40$ & $2.9334 \mathrm{e}-11$ & 6.7413 & $7.9670 \mathrm{e}-11$ & 5.3283
\end{tabular}

Table 6.2: Solution verification and calculation of the convergence rate of $u_{\theta}$ for $1 \mathrm{D}$, two-phase Couette flow without interfacial tension, $\sigma=0$.

\begin{tabular}{c|c|c|c|c} 
& $L_{1}$ error & Order in $L_{1}$ norm & $L_{\infty}$ error & Order in $L_{\infty}$ norm \\
\hline $10 \times 10$ & 0.0025184 & NA & 0.013839 & NA \\
\hline $20 \times 20$ & 0.0011825 & 1.0907 & 0.0089610 & 0.6270 \\
\hline $40 \times 40$ & 0.0005298 & 1.1583 & 0.0025929 & 1.7891
\end{tabular}

\subsection{Couette flow with perturbed interfacial tension $(\sigma=0.001 \mathrm{dyn} / \mathrm{cm})$}

Similarly, for this test with perturbed interfacial tension, errors are computed from $3 \mathrm{D}$ simulations at time $t=28.1 \mathrm{~s}$ ( 1 revolution) and with the reference pressure 
Table 6.3: Solution verification and calculation of the convergence rate of $p$ for $1 \mathrm{D}$, two-phase Couette flow without interfacial tension, $\sigma=0$.

\begin{tabular}{c|c|c|c|c} 
& $L_{1}$ error & Order in $L_{1}$ norm & $L_{\infty}$ error & Order in $L_{\infty}$ norm \\
\hline $10 \times 10$ & 0.0044984 & NA & 0.019847 & NA \\
\hline $20 \times 20$ & 0.0013152 & 1.7741 & 0.0064381 & 1.6242 \\
\hline $40 \times 40$ & 0.0004539 & 1.5348 & 0.0020022 & 1.6850
\end{tabular}

$\Delta p=0.022912 \mathrm{dyn} / \mathrm{cm}^{2}$. The results displayed in tables $6.4,6.5$, and 6.6 are similar to the case with no interfacial tension except for the pressure calculation which does not converge with mesh refinement in the $L_{\infty}$ norm. With a small, non-zero interfacial tension, the analytical solution for pressure becomes discontinuous and this cannot be accommodated by the numerical solution method which approximates the pressure jump with a continuous field.

Table 6.4: Solution verification and calculation of the convergence rate of the interface position for 1D, two-phase Couette flow with perturbed interfacial tension, $\sigma=0.001 \mathrm{dyn} / \mathrm{cm}$.

\begin{tabular}{c|c|c|c|c} 
& $L_{1}$ error & Order in $L_{1}$ norm & $L_{\infty}$ error & Order in $L_{\infty}$ norm \\
\hline $10 \times 10$ & $2.6941 \mathrm{e}-8$ & NA & $2.6951 \mathrm{e}-8$ & NA \\
\hline $20 \times 20$ & $3.1471 \mathrm{e}-9$ & 3.0977 & $3.2094 \mathrm{e}-9$ & 3.0699 \\
\hline $40 \times 40$ & $2.4069 \mathrm{e}-11$ & 7.0307 & $3.2086 \mathrm{e}-11$ & 6.6442
\end{tabular}

Table 6.5: Solution verification and calculation of the convergence rate of $u_{\theta}$ for $1 \mathrm{D}$, two-phase Couette flow with perturbed interfacial tension, $\sigma=0.001 \mathrm{dyn} / \mathrm{cm}$.

\begin{tabular}{c|c|c|c|c} 
& $L_{1}$ error & Order in $L_{1}$ norm & $L_{\infty}$ error & Order in $L_{\infty}$ norm \\
\hline $10 \times 10$ & 0.0025184 & NA & 0.013839 & NA \\
\hline $20 \times 20$ & 0.0011825 & 1.0907 & 0.0089610 & 0.6270 \\
\hline $40 \times 40$ & 0.0005298 & 1.1583 & 0.0025929 & 1.7891
\end{tabular}


Table 6.6: Solution verification and calculation of the convergence rate of $p$ for 1D, two-phase Couette flow with perturbed interfacial tension, $\sigma=0.001 \mathrm{dyn} / \mathrm{cm}$.

\begin{tabular}{c|c|c|c|c} 
& $L_{1}$ error & Order in $L_{1}$ norm & $L_{\infty}$ error & Order in $L_{\infty}$ norm \\
\hline $10 \times 10$ & 0.0041033 & NA & 0.020524 & NA \\
\hline $20 \times 20$ & 0.0013926 & 1.5590 & 0.006899 & 1.5729 \\
\hline $40 \times 40$ & 0.0005164 & 1.4312 & 0.002690 & 1.3588
\end{tabular}

\subsection{Couette flow with interfacial tension $(\sigma=10 \mathrm{dyn} / \mathrm{cm})$}

In this test for a larger interfacial tension, 3D calculations of the error at time $t=$ $28.1 \mathrm{~s}$ ( 1 revolution) and the reference pressure $\Delta p=3.448276 \mathrm{dyn} / \mathrm{cm}$ were obtained. We demonstrate by numerical experiment that the algorithm is first-order accurate

Table 6.7: Solution verification and calculation of the convergence rate of the interface position for 1D, two-phase Couette flow with interfacial tension, $\sigma=10 \mathrm{dyn} / \mathrm{cm}$.

\begin{tabular}{c|c|c|c|c} 
& $L_{1}$ error & Order in $L_{1}$ norm & $L_{\infty}$ error & Order in $L_{\infty}$ norm \\
\hline $10 \times 10$ & $8.2300 \mathrm{e}-7$ & NA & $8.2364 \mathrm{e}-7$ & NA \\
\hline $20 \times 20$ & $9.2962 \mathrm{e}-8$ & 3.1462 & $9.3070 \mathrm{e}-8$ & 3.1456 \\
\hline $40 \times 40$ & $9.3184 \mathrm{e}-9$ & 3.3185 & $9.3199 \mathrm{e}-9$ & 3.3199
\end{tabular}

Table 6.8: Solution verification and calculation of the convergence rate of $u_{\theta}$ for 1D, two-phase Couette flow with interfacial tension, $\sigma=10 \mathrm{dyn} / \mathrm{cm}$.

\begin{tabular}{c|c|c|c|c} 
& $L_{1}$ error & Order in $L_{1}$ norm & $L_{\infty}$ error & Order in $L_{\infty}$ norm \\
\hline $10 \times 10$ & 0.0025164 & NA & 0.0138301 & NA \\
\hline $20 \times 20$ & 0.0011822 & 1.0899 & 0.0089558 & 0.6269 \\
\hline $40 \times 40$ & 0.0005297 & 1.1582 & 0.0025923 & 1.7886
\end{tabular}

for the interface position and velocity field as well as for pressure in an $L_{1}$ error norm. For the $\sigma=10 \mathrm{dyn} / \mathrm{cm}$ interfacial tension case, the $L_{\infty}$ error for pressure does not converge using the continuous surface tension model, which is consistent with the description of IB method in Sections 3.3-3.4. 
Zhou, Ray, Lim, Wang, de Almeida, Glimm, Li, and Jiao

Table 6.9: Solution verification and calculation of the convergence rate of $p$ for $1 \mathrm{D}$, two-phase Couette flow with interfacial tension, $\sigma=10 \mathrm{dyn} / \mathrm{cm}$.

\begin{tabular}{c|c|c|c|c} 
& $L_{1}$ error & Order in $L_{1}$ norm & $L_{\infty}$ error & Order in $L_{\infty}$ norm \\
\hline $10 \times 10$ & 0.0382376 & NA & 0.2708899 & NA \\
\hline $20 \times 20$ & 0.0323036 & 0.2433 & 0.4541237 & -0.7454 \\
\hline $40 \times 40$ & 0.0062075 & 2.3796 & 0.1725304 & 1.3962
\end{tabular}

For future reference and studies, the linear stability theory for two-fluid TaylorCouette flow can be found in (Baier, 1999; Baier and Graham, 1998, 2000). The two fluids are assumed to be stratified in these analyses. Numerical simulations of stratified two-phase Taylor-Couette flow were given in (Vedantam et al., 2006).

\section{Two-phase Couette mixing in a 3-D annular sec- tor}

At a high Reynolds number, a two-phase Couette flow of two immiscible liquid phases with distinct mass density and viscosity will not remain stratified. The phases fully mix by combined shear and centrifugal forces. When the inner cylinder rotates, the heavier fluid is propelled outwards in the direction of the outer cylinder wherein high shear stresses promote vigorous mixing and dispersion formation.

The detailed simulation of a two-phase, liquid-liquid interface evolution in the Couette annulus is a first step towards a fundamental understanding of the micromixing length-scale flow in many annular devices. Here focus is directed to the annular mixing region in a centrifugal contactor. To keep the computational effort at a reasonable cost, a sector of the annulus is selected for analysis by means of periodic boundary conditions in the axial and azimuthal directions. This truncation of the annulus requires the neglect of gravity because of axial periodicity (fig. 7.1). When comparing to a realistic device, this assumption is only plausible in the bulk of the mixing zone where the centrifugal force imparted in the fluid surpasses gravity substantially.

In this study, an interface is positioned within the annulus at a given radial position $R_{\mathrm{I}}$. Accordingly, a heavy liquid (aqueous phase) is assigned to the inner annular region delimited by the interface. A lighter (organic phase), more viscous liquid is placed in the complement outer region of the annulus. This arrangement is unstable with respect to the centrifugal acceleration in the radial outward pointing direction. The initial velocity and pressure fields are set as zero in the fluid ("cold start"), 


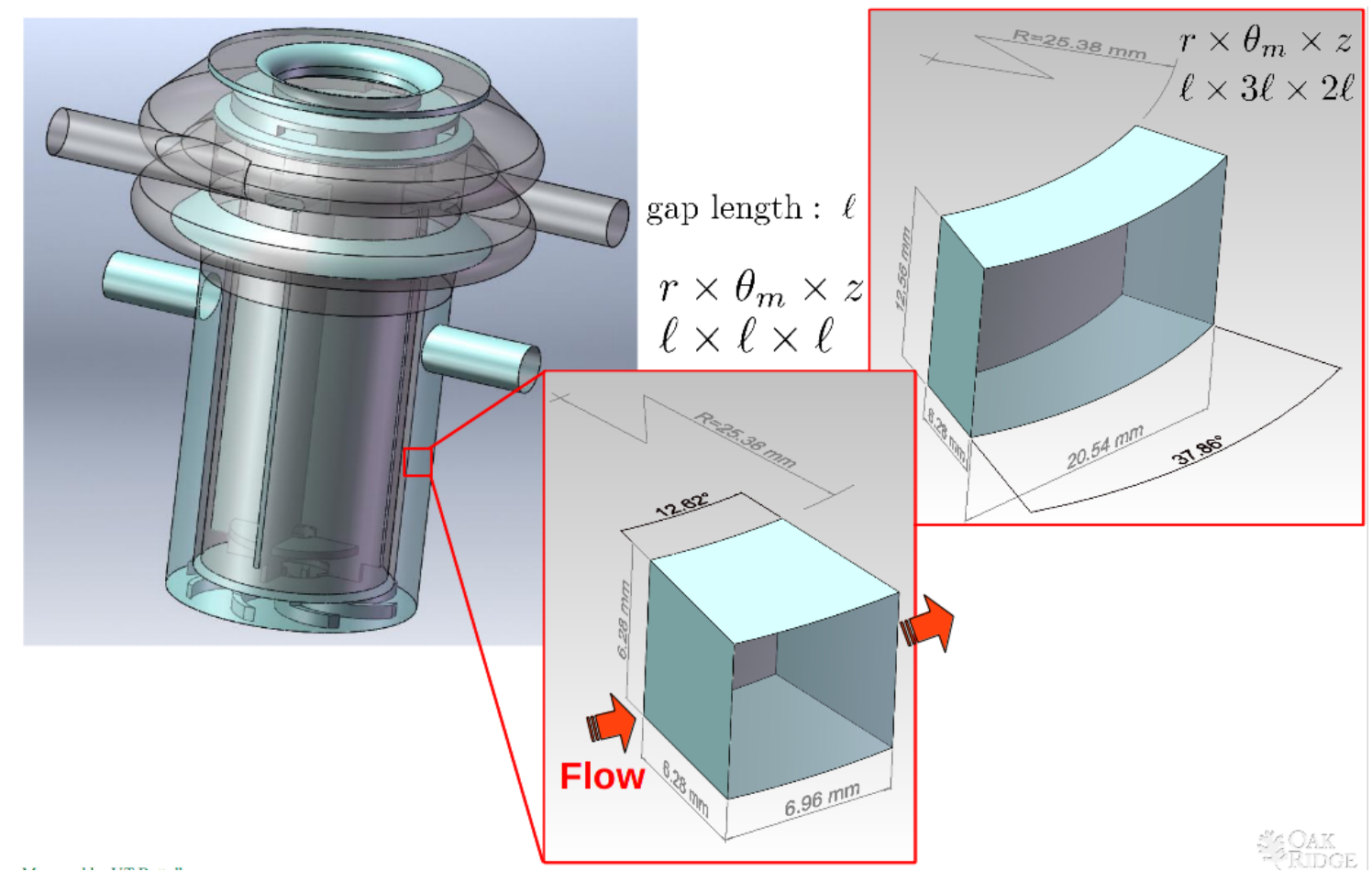

Fig. 7.1: Annular sector of a centrifugal contactor used for the computation domain. On the left is the complete flow domain in a centrifugal contactor. On the center is the smallest computational sector used in this study with one gap length $\ell$ in each direction of the cylindrical coordinates. On the right is a sector with twice the gap length in the axial direction and three times the gap in the azimuth angle direction when evaluated at the average radius. 
and the inner cylinder speed is set at $1500 \mathrm{rpm}$. An alternative initial condition can use the analytical Couette flow fields (6.5)-(6.8). The usage of these different initial conditions is necessary for exploring the onset of chaos as the motion approaches a statistically fully developed regime. Geometrical parameters and physical properties used in this research are collected in Table 7.1.

Table 7.1: Geometric parameters and physical properties for 3D simulation of twophase flow in an annular sector.

\begin{tabular}{|c|c|}
\hline$R_{\mathrm{i}}$ & $2.538 \mathrm{~cm}$ \\
\hline$R_{\mathrm{o}}$ & $3.166 \mathrm{~cm}$ \\
\hline$\ell=R_{\mathrm{o}}-R_{\mathrm{i}}$ & $0.628 \mathrm{~cm}$ \\
\hline$\eta=R_{\mathrm{i}} / R_{\mathrm{o}}$ & 0.8 \\
\hline$\Omega_{\mathrm{i}}$ & $157 \mathrm{radians} / \mathrm{s}(1500 \mathrm{RPM})$ \\
\hline$\mu_{\mathrm{org}}$ & $0.016 \mathrm{~g} / \mathrm{cm} \cdot \mathrm{s}$ \\
\hline$\mu_{\text {aqu }}$ & $0.0102 \mathrm{~g} / \mathrm{cm} \cdot \mathrm{s}$ \\
\hline$\rho_{\text {org }}$ & $0.811 \mathrm{~g} / \mathrm{cm}^{3}$ \\
\hline$\rho_{\text {aqu }}$ & $1.03 \mathrm{~g} / \mathrm{cm}^{3}$ \\
\hline$R e_{\text {org }}:=\frac{\Omega_{\mathrm{i}} R_{\mathrm{i}} \ell}{\nu_{\text {or }} r}$ & $1.27 \times 10^{4}$ \\
\hline$R e_{\text {aqu }}:=\frac{\Omega_{\mathrm{i}} \mathrm{R}_{\mathrm{i}} \ell}{\nu_{\text {aqu }}}$ & $2.53 \times 10^{4}$ \\
\hline$\sigma$ & $10 \mathrm{dyn} / \mathrm{cm}$ \\
\hline
\end{tabular}

The interfacial tension between phases ranges from 10-25 dyn/cm for the intended application. Preliminary results have been obtained for the lower end of the interfacial tension range (fig. 7.2). Here the extension of the domain in the azimuthal direction is $5 \ell$ and in the axial direction is $3 \ell$. An initial, long wavelength, spatial perturbation is added to the position of the interface (fig. 7.2, top-left image). With the motion of the inner cylinder, the aqueous phase moves from the inside region to the outside by centrifugal acceleration displacing the organic phase. The outside annular region applies the greatest shear stress on the fluid therefore promoting mixing of the phases. The deformation of the interface in this application is a challenging phenomenon for scientific modeling since it is driven by the combination of a Kelvin-Helmholtz shear instability and a Rayleigh-Taylor fingering instability. The radially, inward-moving, organic phase is shaped as mushroom caps, similarly for the aqueous phase moving in the opposite direction (fig. 7.2). The caps penetrate the opposing fluid phase and are sheared by the velocity gradient across the annular gap (fig. 7.2, right column at 47 ms starting from a "cold start").

By virtue of incompressibility the volume ratio of the organic to aqueous phase

Oak Ridge National Laboratory Technical Report ORNL/TM-2012/28 — pp. 1-55 

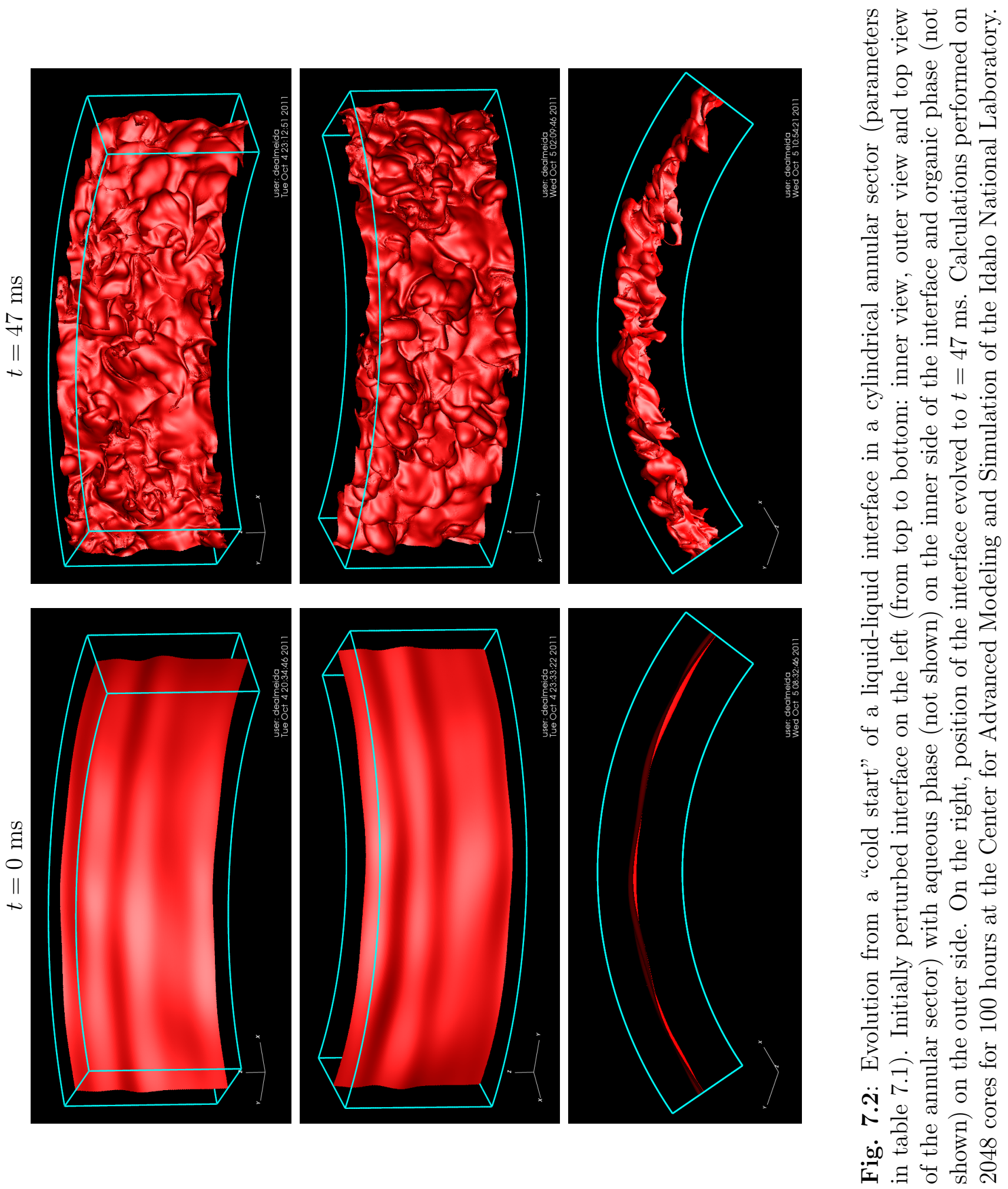
$\left(\phi_{\mathrm{O} / \mathrm{A}}:=\frac{V_{\mathrm{O}}}{V_{\mathrm{A}}}=0.82\right)$ is an invariant; so is each individual phase volume. Therefore the stretching and break up of the interface must preserve the initial volume of each phase for truly incompressible fluids. In this work, the volume-conserving constraint hinges upon the interpolant (3.1) as applied to the mass density and dynamic viscosity fields (3.2)-(3.3). This interpolant it is not necessarily compatible in the sense of (2.9)-(2.10). However we monitor our calculations for the departure from a true incompressible fluid behavior (fig. 7.3) which is insignificant for the initial stages of mixing.

A quantity of great interest is the growth of the interface area with time. The principal objective of mixing is to increase the interfacial area to a maximum. Here we compute this quantity which demonstrate exponential growth while preserving volume (fig. 7.3). These results are preliminary and mixing has not reached a statistically fully developed regime. This is the subject of current and future developments. Evolution of the mixing flow to a point where the interfacial area reaches a mean value is a challenging problem not addressed by previous researchers.

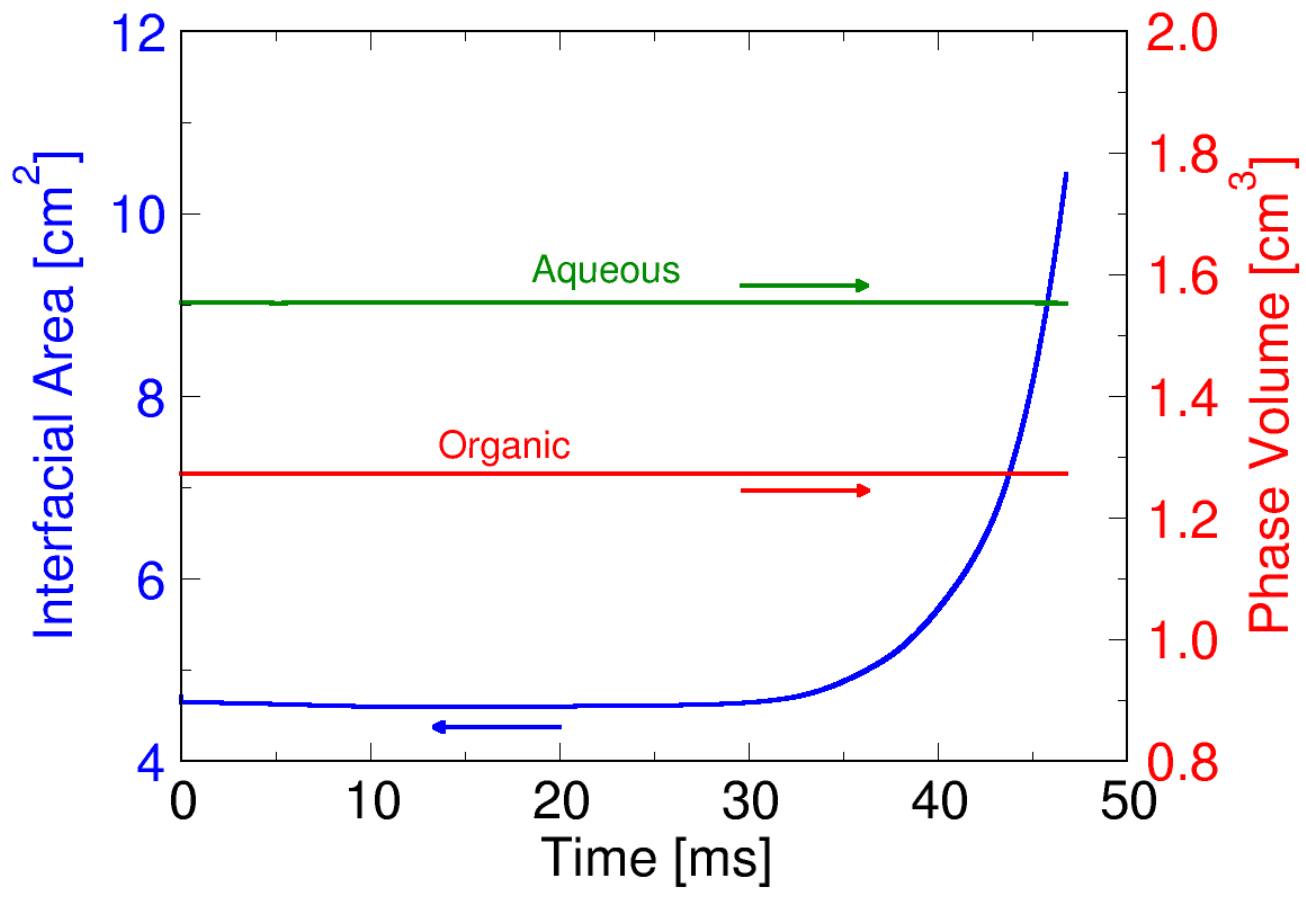

Fig. 7.3: Growth of interfacial area and conservation of volume for an initial stage of micromixing (fig. 7.2). Calculations performed on 2048 cores for 100 hours at the Center for Advanced Modeling and Simulation of the Idaho National Laboratory. Spatial mesh $128 \times \times 256 \times 512$ in the $r \times \theta \times z$ directions. 


\section{Summary and outlook}

Immiscible, liquid-liquid mixing in solvent extraction processes is vigorous and therefore complex. The central objective is to promote chemical transport from one liquid phase to the other via the creation of an extensive interfacial area. This work is prompted by the need to predict, at the micrometer length scale, the interfacial area by evolving a mathematical surface in space and time with precision never done before in this application area.

Accomplishment of this task will enable the development of coarsening theories for modeling the macroscopic transport of chemical species at the contactor device scale used in solvent extraction. This undertaking is not part of the current scope of work.

A front tracking approach has been taken in this work for solving the two-phase, microfluidics equations of motion for Newtonian fluids. Progressive verification and validation, to the extent possible, have been described here with satisfactory success. The test problem used is an annular sector of a centrifugal contactor; a common mixing device used in solvent extraction processes.

The front tracking simulation capability is in ongoing development with additional improvements for advancing simulations to a fully developed mixing regime and characterization of the flow structure in terms of dispersed and continuous fluid phases. A final report on these advances will be issued in the future. 


\section{A Equations of motion for incompressible Newto- nian fluids}

\section{A.1 One-phase fluid flow}

For a single-phase incompressible fluid with constant density and viscosity, the incompressible Navier-Stokes equations along with the equation of continuity in cylindrical coordinates are

$$
\begin{aligned}
& \frac{1}{r} \frac{\partial u_{\theta}}{\partial \theta}+\frac{\partial u_{z}}{\partial z}+\frac{1}{r} \frac{\partial\left(r u_{r}\right)}{\partial r}=0 \\
& \rho\left(\frac{\partial u_{\theta}}{\partial t}+\frac{u_{\theta}}{r} \frac{\partial u_{\theta}}{\partial \theta}+u_{z} \frac{\partial u_{\theta}}{\partial z}+u_{r} \frac{\partial u_{\theta}}{\partial r}+\frac{u_{r} u_{\theta}}{r}\right) \\
& =-\frac{1}{r} \frac{\partial p}{\partial \theta}+\mu\left[\frac{1}{r^{2}} \frac{\partial^{2} u_{\theta}}{\partial \theta^{2}}+\frac{\partial^{2} u_{\theta}}{\partial z^{2}}+\frac{1}{r} \frac{\partial}{\partial r}\left(r \frac{\partial u_{\theta}}{\partial r}\right)-\frac{u_{\theta}}{r^{2}}+\frac{2}{r^{2}} \frac{\partial u_{r}}{\partial \theta}\right]+\rho f_{\theta} \\
& \rho\left(\frac{\partial u_{z}}{\partial t}+\frac{u_{\theta}}{r} \frac{\partial u_{z}}{\partial \theta}+u_{z} \frac{\partial u_{z}}{\partial z}+u_{r} \frac{\partial u_{z}}{\partial r}\right) \\
& =-\frac{\partial p}{\partial z}+\mu\left[\frac{1}{r^{2}} \frac{\partial^{2} u_{z}}{\partial \theta^{2}}+\frac{\partial^{2} u_{z}}{\partial z^{2}}+\frac{1}{r} \frac{\partial}{\partial r}\left(r \frac{\partial u_{z}}{\partial r}\right)\right]+\rho f_{z} \\
& \rho\left(\frac{\partial u_{r}}{\partial t}+\frac{u_{\theta}}{r} \frac{\partial u_{r}}{\partial \theta}+u_{z} \frac{\partial u_{r}}{\partial z}+u_{r} \frac{\partial u_{r}}{\partial r}-\frac{u_{\theta} u_{\theta}}{r}\right) \\
& =-\frac{\partial p}{\partial r}+\mu\left[\frac{1}{r^{2}} \frac{\partial^{2} u_{r}}{\partial \theta^{2}}+\frac{\partial^{2} u_{r}}{\partial z^{2}}+\frac{1}{r} \frac{\partial}{\partial r}\left(r \frac{\partial u_{r}}{\partial r}\right)-\frac{u_{r}}{r^{2}}-\frac{2}{r^{2}} \frac{\partial u_{\theta}}{\partial \theta}\right]+\rho f_{r} .
\end{aligned}
$$

\section{A.2 Axisymmetric linear stability analysis of Couette flow}

The transition from laminar Couette flow to Taylor vortex flow occurs at the critical Reynolds number 94.7 according to a classical linear stability analysis of small perturbations. The governing equations for the evolution of these normal mode disturbances as applied to the base Couette flow are presented here for completeness of this report and as background for Section 5.3. Infinitesimal disturbances are applied to the Couette flow in the domain

$$
R_{1}<r<R_{2}, 0 \leq \theta<2 \pi \text { and }-\infty<z<\infty,
$$

with an aspect ratio $\Gamma=\infty$.

The velocity field for the Couette flow in cylindrical coordinates is given by

$$
v_{r}=v_{z}=0, v_{\theta}=V(r)=A r+\frac{B}{r}, \frac{\partial p}{\partial r}=\rho \frac{V^{2}(r)}{r}
$$


where $\rho$ is the density and

$$
\begin{aligned}
& A=\frac{R_{2}^{2} \Omega_{2}-R_{1}^{2} \Omega_{1}}{R_{2}^{2}-R_{1}^{2}}=-\Omega_{1} \frac{\eta^{2}-\mu}{1-\eta^{2}} \\
& B=-\frac{R_{1}^{2} R_{2}^{2}\left(\Omega_{2}-\Omega_{1}\right)}{R_{2}^{2}-R_{1}^{2}}=\Omega_{1} R_{1}^{2} \frac{1-\mu}{1-\eta^{2}} \\
& \mu=\frac{\Omega_{2}}{\Omega_{1}}, \eta=\frac{R_{1}}{R_{2}} .
\end{aligned}
$$

The Couette flow can be characterized by the parameters $\mu$, $\eta$, and Reynolds number. Here the values for the parameters are

$$
\Omega_{2}=0, \mu=0, \eta=\frac{R_{1}}{R_{2}}=\frac{2.538}{3.166}=0.8 .
$$

Let $u^{\prime}, v^{\prime}, w^{\prime}$ and $p^{\prime}$ denote the perturbations of $v_{r}, v_{\theta}, v_{z}$ and $p$ for Couette flow. Substituting $v_{r} \rightarrow v_{r}+u^{\prime}=u^{\prime}, v_{\theta} \rightarrow v_{\theta}+v^{\prime}=V+v^{\prime}, v_{z} \rightarrow v_{z}+w^{\prime}=w^{\prime}$ and $p \rightarrow p+p^{\prime}$ in the Navier-Stokes equations (A.1), we get

$$
\begin{aligned}
& r: \frac{\partial u^{\prime}}{\partial t}+\frac{V}{r} \frac{\partial u^{\prime}}{\partial \theta}-\frac{2 V}{r} v^{\prime}+\frac{1}{\rho} \frac{\partial p^{\prime}}{\partial r}-\nu\left(\nabla^{2} u^{\prime}-\frac{2}{r^{2}} \frac{\partial v^{\prime}}{\partial \theta}-\frac{u^{\prime}}{r^{2}}\right) \\
& =-\left(u^{\prime} \frac{\partial u^{\prime}}{\partial r}+\frac{v^{\prime}}{r} \frac{\partial u^{\prime}}{\partial \theta}+w^{\prime} \frac{\partial u^{\prime}}{\partial z}\right)+\frac{v^{\prime 2}}{r} \\
& \theta: \frac{\partial v^{\prime}}{\partial t}+\frac{d V}{d r} u^{\prime}+\frac{V}{r} \frac{\partial v^{\prime}}{\partial \theta}+\frac{V}{r} u^{\prime}+\frac{1}{\rho r} \frac{\partial p^{\prime}}{\partial \theta}-\nu\left(\nabla^{2} v^{\prime}+\frac{2}{r^{2}} \frac{\partial u^{\prime}}{\partial \theta}-\frac{v^{\prime}}{r^{2}}\right) \\
& =-\left(u^{\prime} \frac{\partial v^{\prime}}{\partial r}+\frac{v^{\prime}}{r} \frac{\partial v^{\prime}}{\partial \theta}+w^{\prime} \frac{\partial v^{\prime}}{\partial z}\right)-\frac{v^{\prime} u^{\prime}}{r} \\
& z: \frac{\partial w^{\prime}}{\partial t}+\frac{V}{r} \frac{\partial w^{\prime}}{\partial \theta}+\frac{1}{\rho} \frac{\partial p^{\prime}}{\partial z}-\nu \nabla^{2} w^{\prime}=-\left(u^{\prime} \frac{\partial w^{\prime}}{\partial r}+\frac{v^{\prime}}{r} \frac{\partial w^{\prime}}{\partial \theta}+w^{\prime} \frac{\partial w^{\prime}}{\partial z}\right) .
\end{aligned}
$$

Similarly for the equation of continuity

$$
\frac{\partial u^{\prime}}{\partial r}+\frac{u^{\prime}}{r}+\frac{1}{r} \frac{\partial v^{\prime}}{\partial \theta}+\frac{\partial w^{\prime}}{\partial z}=0 .
$$

The no slip condition at the inner and outer cylinder gives the boundary conditions

$$
u^{\prime}=v^{\prime}=w^{\prime}=0 \text { at } r=R_{1} \text { and } r=R_{2} .
$$

We consider infinitesimal disturbances that are axisymmetric, i.e. independent of $\theta$, and periodic in the axial direction. Linearizing the equations (A.12), (A.13), (A.14) 
and (A.15) and taking into account that all the disturbances are independent of $\theta$ we obtain

$$
\begin{array}{r}
\frac{\partial u^{\prime}}{\partial t}-\frac{2 V}{r} v^{\prime}=-\frac{1}{\rho} \frac{\partial p^{\prime}}{\partial r}+\nu\left(\nabla^{2} u^{\prime}-\frac{u^{\prime}}{r^{2}}\right) \\
\frac{\partial v^{\prime}}{\partial t}+\left(\frac{d V}{d r}+\frac{V}{r}\right) u^{\prime}=\nu\left(\nabla^{2} v^{\prime}-\frac{v^{\prime}}{r^{2}}\right) \\
\frac{\partial w^{\prime}}{\partial t}=-\frac{1}{\rho} \frac{\partial p^{\prime}}{\partial z}+\nu \nabla^{2} w^{\prime} \\
\frac{\partial u^{\prime}}{\partial r}+\frac{u^{\prime}}{r}+\frac{\partial w^{\prime}}{\partial z}=0
\end{array}
$$

where $\nabla^{2}=\frac{\partial^{2}}{\partial r^{2}}+\frac{1}{r} \frac{\partial}{\partial r}+\frac{\partial^{2}}{\partial z^{2}}$.

The normal mode solutions for the disturbances have the form

$$
u^{\prime}=u(r) e^{\beta t+i \lambda z}, v^{\prime}=v(r) e^{\beta t+i \lambda z}, w^{\prime}=w(r) e^{\beta t+i \lambda z}, p^{\prime}=P(r) e^{\beta t+i \lambda z} .
$$

In these disturbances $\beta$ is the growth rate and $\lambda$ is the wavenumber in the axial direction. Substituting these expressions in (A.17), (A.18), (A.19) and (A.20) we get

$$
\begin{array}{r}
\nu\left(\frac{d^{2}}{d r^{2}}+\frac{1}{r} \frac{d}{d r}-\frac{1}{r^{2}}-\lambda^{2}-\frac{\beta}{\nu}\right) u=\frac{1}{\rho} \frac{d P}{d r}-\frac{2 V}{r} v \\
\nu\left(\frac{d^{2}}{d r^{2}}+\frac{1}{r} \frac{d}{d r}-\frac{1}{r^{2}}-\lambda^{2}-\frac{\beta}{\nu}\right) v=2 A u \\
\nu\left(\frac{d^{2}}{d r^{2}}+\frac{1}{r} \frac{d}{d r}-\lambda^{2}-\frac{\beta}{\nu}\right) w=\frac{1}{\rho} P(i \lambda z) \\
\frac{d u}{d r}+\frac{u}{r}+i \lambda w=0 .
\end{array}
$$

Finally, eliminating $P$ and $w$ we obtain

$$
\begin{array}{r}
\nu\left(\frac{d^{2}}{d r^{2}}+\frac{1}{r} \frac{d}{d r}-\frac{1}{r^{2}}-\lambda^{2}-\frac{\beta}{\nu}\right)\left(\frac{d^{2}}{d r^{2}}+\frac{1}{r} \frac{d}{d r}-\frac{1}{r^{2}}-\lambda^{2}\right) u=2 \lambda^{2} \Omega(r) v \\
\nu\left(\frac{d^{2}}{d r^{2}}+\frac{1}{r} \frac{d}{d r}-\frac{1}{r^{2}}-\lambda^{2}-\frac{\beta}{\nu}\right) v=2 A u
\end{array}
$$

where $\Omega(r)=\frac{V}{r}$. From (A.16) we get at $r=R_{1}$ and $r=R_{2}$

$$
u=v=0 \text {. }
$$

From equation of continuity we get at $r=R_{1}$ and $r=R_{2}$

$$
\frac{d u}{d r}=0
$$


Using the transformations (Prima and Swinney, 1985):

$$
\begin{aligned}
r & =R_{0}+d x, R_{0}=\frac{R_{1}+R_{2}}{2} \\
\delta & =\frac{d}{R_{0}}, \xi(x)=\frac{1}{1+\delta x} \\
\Omega & =\Omega_{1} G(x), G(x)=\frac{1}{1-\eta^{2}}\left[\mu-\eta^{2}+4 \xi^{2}(x) \eta^{2} \frac{1-\mu}{(1+\eta)^{2}}\right] \\
\sigma & =\frac{\beta d^{2}}{\nu}, \alpha=\lambda d, T=-\frac{4 A \Omega_{1} d^{4}}{\nu^{2}} \\
D & =\frac{d}{d x}, D^{*}=D+\delta \xi(x) .
\end{aligned}
$$

on equations (A.26) and (A.27), we obtain

$$
\begin{aligned}
& \left(D D^{*}-\alpha^{2}-\sigma\right)\left(D D^{*}-\alpha^{2}\right) u_{11}=-\alpha^{2} T G(x, \mu, \eta) v_{11}, \\
& \left(D D^{*}-\alpha^{2}-\sigma\right)=u_{11} .
\end{aligned}
$$

with the boundary conditions

$$
u_{11}=v_{11}=D u_{11}=0 \text { at } x= \pm \frac{1}{2} .
$$

Equations (A.32)-(A.34) form an eigenvalue problem in two parameters along with the boundary conditions. The flow can be described in terms of the geometry parameter $\eta$, the fluid parameters ( $\mu$ and Taylor's number $T$ ) and the disturbance parameters $\alpha$ and $\sigma$. It can be shown that for given $\mu$ and $\eta$ there exists a value $T_{c}$ such that for $T<T_{c}, \operatorname{Re}(\sigma)<0$ for all $\alpha>0$ and for $T>T_{c}, \operatorname{Re}(\sigma)>0$ for a band of wave numbers $\left(\alpha_{-}<\alpha<\alpha_{+}\right)$. This defines a set of points in $T, \alpha$ space which is called the neutral curve (see fig. A.1) . Clearly the minimum point of the neutral curve is the $T_{c}$ below which all disturbances of any wavenumber decay with time and above which there is a band of wavenumbers for which the disturbances grow exponentially with time. Table A.1 shows the critical Reynolds and wavenumbers for different radius ratios $\eta$ (Chandrasekhar, 1961; Prima and Swinney, 1985; Roberts, 1965; Sparrow et al., 1974; Walowit et al., 1964). At marginal stability the growth rate of the critical wavenumber is zero $(\sigma=0)$. For a supercritical disturbance the growth rate will be positive. From linearized theory, the disturbance grows exponentially with time. However, the disturbances do not show continual amplification with time and instead attains a finite equilibrium amplitude.

Davey (1962); Wereley and Lueptow (1994) showed that the growth rate is proportional to the reduced Reynolds number $\varepsilon=1-\frac{R_{c}^{2}}{R e^{2}}$. He also calculated the coefficient 
Table A.1: Critical Reynolds number $(\Gamma=\infty, \mu=0)$ of axisymmetric, linear stability analysis.

\begin{tabular}{c|c|c}
$\eta$ & $R e_{\text {crit }}$ & $k_{\text {crit }}$ \\
\hline 0.975 & 260.9 & 3.13 \\
\hline 0.90 & 131.6 & 3.13 \\
\hline 0.80 & 94.7 & 3.13 \\
\hline 0.70 & 79.5 & 3.14 \\
\hline 0.60 & 71.7 & 3.15 \\
\hline 0.50 & 68.2 & 3.16
\end{tabular}

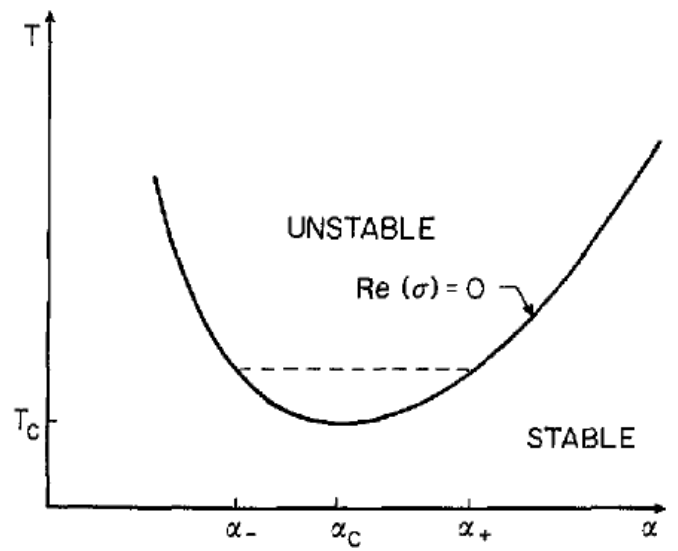

Fig. A.1: Neutral curve of axisymmetric, linear stability analysis (Prima and Swinney, 1985). 
of the reduced Reynolds number for the growth rate for the wide gap case $\left(R_{2}=2 R_{1}\right)$ and the narrow gap case $(\eta \rightarrow 1)$ (Davey, 1962).

\section{A.3 Two-phase fluid flow}

As we described in Sec. 3.4, we smooth the density and viscosity and spread out the singular force into a strip when using IB. Thus at each step, the incompressible Navier-Stokes equations with variable density and viscosity need to be solved.

In rectangular coordinate, these equations become

$$
\begin{aligned}
& \frac{\partial u}{\partial x}+\frac{\partial v}{\partial y}=0 \\
& u_{t}+u \frac{\partial u}{\partial x}+v \frac{\partial u}{\partial y}=-\frac{p_{x}}{\rho}+\frac{\left(2 \mu u_{x}\right)_{x}+\left(\mu\left(u_{y}+v_{x}\right)\right)_{y}}{\rho}+F_{x}+G_{x} \\
& v_{t}+u \frac{\partial v}{\partial x}+v \frac{\partial v}{\partial y}=-\frac{p_{y}}{\rho}+\frac{\left(\mu\left(u_{y}+v_{x}\right)\right)_{x}+\left(2 \mu v_{y}\right)_{y}}{\rho}+F_{y}+G_{y}
\end{aligned}
$$

in $2 \mathrm{D}$. In $3 \mathrm{D}$ they take the form

$$
\begin{aligned}
& \frac{\partial u}{\partial x}+\frac{\partial v}{\partial y}+\frac{\partial w}{\partial z}=0 \\
& u_{t}+u \frac{\partial u}{\partial x}+v \frac{\partial u}{\partial y}+w \frac{\partial u}{\partial z} \\
& =-\frac{p_{x}}{\rho}+\frac{\left(2 \mu u_{x}\right)_{x}+\left(\mu\left(u_{y}+v_{x}\right)\right)_{y}+\left(\mu\left(u_{z}+w_{x}\right)\right)_{z}}{\rho}+F_{x}+G_{x} \\
& v_{t}+u \frac{\partial v}{\partial x}+v \frac{\partial v}{\partial y}+w \frac{\partial v}{\partial z} \\
& =-\frac{p_{y}}{\rho}+\frac{\left(\mu\left(u_{y}+v_{x}\right)\right)_{x}+\left(2 \mu v_{y}\right)_{y}+\left(\mu\left(v_{z}+w_{y}\right)\right)_{z}}{\rho}+F_{y}+G_{y} \\
& w_{t}+u \frac{\partial w}{\partial x}+v \frac{\partial w}{\partial y}+w \frac{\partial w}{\partial z} \\
& =-\frac{p_{z}}{\rho}+\frac{\left(\mu\left(u_{z}+w\right)_{x}+\left(\mu\left(v_{z}+w_{y}\right)\right)_{y}+\left(2 \mu w_{z}\right)_{z}\right.}{\rho}+F_{z}+G_{z}
\end{aligned}
$$


Transforming into cylindrical coordinates, we have

$$
\begin{aligned}
& \frac{\partial u_{z}}{\partial z}+\frac{\partial u_{r}}{\partial r}+\frac{1}{r} \frac{\partial u_{\theta}}{\partial \theta}+\frac{u_{r}}{r}=0 \\
& \frac{\partial u_{\theta}}{\partial t}+\frac{\partial\left(u_{z} u_{\theta}\right)}{\partial z}+\frac{\partial\left(u_{r} u_{\theta}\right)}{\partial r}+\frac{1}{r} \frac{\partial\left(u_{\theta} u_{\theta}\right)}{\partial \theta}+2 \frac{u_{r} u_{\theta}}{r} \\
& =\frac{1}{\rho}\left[-\frac{1}{r} \frac{\partial p}{\partial \theta}+\frac{\partial d_{z \theta}}{\partial z}+\frac{\partial d_{r \theta}}{\partial r}+\frac{1}{r} \frac{\partial d_{\theta \theta}}{\partial \theta}+2 \frac{\left.d_{r \theta}\right]}{r}\right]+F_{\theta}+G_{\theta}, \\
& \frac{\partial u_{z}}{\partial t}+\frac{\partial\left(u_{z} u_{z}\right)}{\partial z}+\frac{\partial\left(u_{r} u_{z}\right)}{\partial r}+\frac{1}{r} \frac{\partial\left(u_{\theta} u_{z}\right)}{\partial \theta}+\frac{u_{r} u_{z}}{r} \\
& =\frac{1}{\rho}\left[-\frac{\partial p}{\partial z}+\frac{\partial d_{z z}}{\partial z}+\frac{\partial d_{r z}}{\partial r}+\frac{1}{r} \frac{\partial d_{\theta z}}{\partial \theta}+\frac{d_{r z}}{r}\right]+F_{z}+G_{z}, \\
& \frac{\partial u_{r}}{\partial t}+\frac{\partial\left(u_{z} u_{r}\right)}{\partial z}+\frac{\partial\left(u_{r} u_{r}\right)}{\partial r}+\frac{1}{r} \frac{\partial\left(u_{\theta} u_{r}\right)}{\partial \theta}+\frac{\left(u_{r} u_{r}-u_{\theta} u_{\theta}\right)}{r} \\
& =\frac{1}{\rho}\left[-\frac{\partial p}{\partial r}+\frac{\partial d_{z r}}{\partial z}+\frac{\partial d_{r r}}{\partial r}+\frac{1}{r} \frac{\partial d_{\theta r}}{\partial \theta}+\frac{\left(d_{r r}-d_{\theta \theta}\right)}{r}\right]+F_{r}+G_{r}
\end{aligned}
$$

where the components of the viscous tensor, $d_{i j}(i, j=\theta, z, r)$ are given by:

$$
\begin{aligned}
& d_{z z}=2 \mu\left(\frac{\partial u_{z}}{\partial z}\right), d_{r r}=2 \mu\left(\frac{\partial u_{r}}{\partial r}\right), d_{\theta \theta}=2 \mu\left(\frac{1}{r} \frac{\partial u_{\theta}}{\partial \theta}+\frac{u_{r}}{r}\right), \\
& d_{z r}=d_{r z}=\mu\left(\frac{\partial u_{r}}{\partial z}+\frac{\partial u_{z}}{\partial r}\right), \\
& d_{z \theta}=d_{\theta z}=\mu\left(\left(\frac{\partial u_{\theta}}{\partial z}+\frac{1}{r} \frac{\partial u_{z}}{\partial \theta}\right),\right. \\
& d_{r \theta}=d_{\theta r}=\mu\left(\frac{\partial u_{\theta}}{\partial r}-\frac{u_{\theta}}{r}+\frac{1}{r} \frac{\partial u_{r}}{\partial \theta}\right) .
\end{aligned}
$$

with $\mu$ as the dynamic viscosity.

\section{A.4 Large-eddy filtered equations for one-phase fluid flow}

The incompressible, LES-filtered Navier-Stokes equations in cylindrical coordinates are presented below. The low-pass filtered quantities are considered to be mesh cell 
averages and denoted with an overbar. Repeated indices are summed.

$$
\begin{aligned}
& \frac{\partial \bar{u}_{z}}{\partial z}+\frac{\partial \bar{u}_{r}}{\partial r}+\frac{1}{r} \frac{\partial \bar{u}_{\theta}}{\partial \theta}+\frac{\bar{u}_{r}}{r}=0, \\
& \frac{\partial \bar{u}_{\theta}}{\partial t}+\frac{\partial \bar{u}_{z} \bar{u}_{\theta}}{\partial z}+\frac{\partial \bar{u}_{r} \bar{u}_{\theta}}{\partial r}+\frac{1}{r} \frac{\partial \bar{u}_{\theta} \bar{u}_{\theta}}{\partial \theta}+2 \frac{\bar{u}_{r} \bar{u}_{\theta}}{r} \\
& =\frac{1}{\bar{\rho}}\left(-\frac{1}{r} \frac{\partial \bar{p}}{\partial \theta}+\frac{1}{r} \frac{\partial \overline{d_{\theta \theta}}}{\partial \theta}+\frac{\partial \overline{d_{z \theta}}}{\partial z}+\frac{\partial \overline{d_{r \theta}}}{\partial r}+2 \frac{\overline{d_{r \theta}}}{r}\right)-\frac{1}{r} \frac{\partial \tau_{\theta \theta}}{\partial \theta}-\frac{\partial \tau_{z \theta}}{\partial z}-\frac{\partial \tau_{r \theta}}{\partial r}-2 \frac{\tau_{r \theta}}{r}+\bar{f}_{\theta}, \\
& \frac{\partial \bar{u}_{z}}{\partial t}+\frac{\partial \bar{u}_{z} \bar{u}_{z}}{\partial z}+\frac{\partial \bar{u}_{r} \bar{u}_{z}}{\partial r}+\frac{1}{r} \frac{\partial \bar{u}_{\theta} \bar{u}_{z}}{\partial \theta}+\frac{\partial \bar{u}_{r} \bar{u}_{z}}{r} \\
& =\frac{1}{\bar{\rho}}\left(-\frac{\partial \bar{p}}{\partial z}+\frac{1}{r} \frac{\partial \overline{d_{\theta z}}}{\partial \theta}+\frac{\partial \overline{d_{z z}}}{\partial z}+\frac{\partial \overline{d_{r z}}}{\partial r}+\frac{\overline{d_{r z}}}{r}\right)-\frac{1}{r} \frac{\partial \tau_{\theta z}}{\partial \theta}-\frac{\partial \tau_{z z}}{\partial z}-\frac{\partial \tau_{r z}}{\partial r}-\frac{\tau_{r z}}{r}+\bar{f}_{z}, \\
& \frac{\partial \bar{u}_{r}}{\partial t}+\frac{\partial \bar{u}_{z} \bar{u}_{r}}{\partial z}+\frac{\partial \bar{u}_{r} \bar{u}_{r}}{\partial r}+\frac{1}{r} \frac{\partial \bar{u}_{\theta} \bar{u}_{r}}{\partial \theta}+\frac{\bar{u}_{r} \bar{u}_{r}-\bar{u}_{\theta} \bar{u}_{\theta}}{r} \\
& =\frac{1}{\bar{\rho}}\left(-\frac{\partial \bar{p}}{\partial r}+\frac{1}{r} \frac{\partial \bar{d}_{\theta r}}{\partial \theta}+\frac{\partial \overline{d_{z r}}}{\partial z}+\frac{\partial \overline{d_{r r}}}{\partial r}+\frac{\overline{d_{r r}}-\overline{d_{\theta \theta}}}{r}\right)-\frac{1}{r} \frac{\partial \tau_{\theta r}}{\partial \theta}-\frac{\partial \tau_{z r}}{\partial z}-\frac{\partial \tau_{r r}}{\partial r}-\frac{\tau_{r r}-\tau_{\theta \theta}}{r}+\bar{f}_{r} .
\end{aligned}
$$

Where $\bar{u}_{\theta}, \bar{u}_{z}$ and $\bar{u}_{r}$ are the velocity components in the azimuthal, axial and radial directions, respectively, and $\bar{\rho}$ and $\bar{p}$ represent the density and the pressure, and the $\bar{f}_{i}(i=\theta, z, r)$ denote body forces.

The components of the viscous stress tensor $\overline{d_{i j}}(i, j=\theta, z, r)$ are given by

$$
\begin{gathered}
\overline{d_{\theta \theta}}=2 \bar{\mu}\left(\frac{1}{r} \frac{\partial \bar{u}_{\theta}}{\partial \theta}+\frac{\bar{u}_{r}}{r}\right), \quad \overline{d_{z z}}=2 \bar{\mu} \frac{\partial \bar{u}_{z}}{\partial z}, \quad \overline{d_{r r}}=2 \bar{\mu} \frac{\partial \bar{u}_{r}}{\partial r}, \\
\overline{d_{r \theta}}=\bar{\mu}\left(\frac{\partial \bar{u}_{\theta}}{\partial r}+\frac{1}{r} \frac{\partial \bar{u}_{r}}{\partial \theta}-\frac{\bar{u}_{\theta}}{r}\right), \quad \overline{d_{\theta z}}=\bar{\mu}\left(\frac{\partial \bar{u}_{\theta}}{\partial z}+\frac{1}{r} \frac{\partial \bar{u}_{z}}{\partial \theta}\right), \quad \overline{d_{z r}}=\bar{\mu}\left(\frac{\partial \bar{u}_{r}}{\partial z}+\frac{\partial \bar{u}_{z}}{\partial r}\right) .
\end{gathered}
$$

The subgrid scale (SGS) variables $\tau_{i j}(i, j=\theta, z, r)$ are defined by

$$
\tau_{i j}=\overline{u_{i} u_{j}}-\bar{u}_{i} \bar{u}_{j} .
$$

We use the dynamic eddy viscosity model (Germano et al., 1991; Ma, 2006) for $\tau_{i j}$. The anisotropic part of $\tau_{i j}$ is modeled as

$$
\tau_{i j}^{M}=\tau_{i j}-\frac{\delta_{i j}}{3} \tau_{k k}=-2 C_{S} \Delta^{2}|\bar{S}| \bar{S}_{i j},
$$


where

$$
\begin{gathered}
\bar{S}_{\theta \theta}=\frac{1}{r} \frac{\partial \bar{u}_{\theta}}{\partial \theta}+\frac{\bar{u}_{r}}{r}, \quad \bar{S}_{z z}=\frac{\partial \bar{u}_{z}}{\partial z}, \quad \bar{S}_{r r}=\frac{\partial \bar{u}_{r}}{\partial r} \\
\bar{S}_{r \theta}=\frac{1}{2}\left(\frac{\partial \bar{u}_{\theta}}{\partial r}+\frac{1}{r} \frac{\partial \bar{u}_{r}}{\partial \theta}-\frac{\bar{u}_{\theta}}{r}\right), \quad \bar{S}_{\theta z}=\frac{1}{2}\left(\frac{\partial \bar{u}_{\theta}}{\partial z}+\frac{1}{r} \frac{\partial \bar{u}_{z}}{\partial \theta}\right), \quad \bar{S}_{z r}=\frac{1}{2}\left(\frac{\partial \bar{u}_{r}}{\partial z}+\frac{\partial \bar{u}_{z}}{\partial r}\right) .
\end{gathered}
$$

and

$$
|\bar{S}|^{2}=\sum 2 S_{i j}^{2}
$$

The $C_{S}$ is a model coefficient to be computed dynamically. Let a spatially test-filtered quantity be denoted by a caret. The test filtered stress $T_{i j}$ is defined by

$$
T_{i j}=\widehat{\overline{u_{i} u_{j}}}-\widehat{\overline{u_{i}}} \widehat{\overline{u_{j}}}
$$

and the anisotropic part of $T_{i j}$ is modeled as

$$
T_{i j}^{M}=-2 C_{S} \widehat{\Delta}^{2} \mid \widehat{\bar{S}}^{\mid} \widehat{\bar{S}}_{i j}
$$

From the Germano's identity,

$$
L_{i j}=T_{i j}-\widehat{\tau_{i j}}=\widehat{\bar{u}_{i} \bar{u}_{j}}-\widehat{\overline{u_{i}}} \widehat{\overline{u_{j}}}
$$

where $L_{i j}$ is the Leonard stress. The right hand side is completely computable from the resolved variables. In addition,

$$
L_{i j}^{a}=T_{i j}^{M}-\widehat{\tau_{i j}^{M}}=2 C_{S} \Delta^{2}\left|\widehat{\bar{S} \mid \bar{S}_{i j}}-2 C_{S} \widehat{\Delta}^{2}\right| \widehat{\bar{S}} \mid \widehat{\bar{S}}_{i j}=C_{S} M_{i j}
$$

where

$$
M_{i j}=2 \Delta^{2}\left|\widehat{\bar{S}}_{i j}-2 \widehat{\Delta}^{2}\right| \widehat{\bar{S}} \mid \widehat{\bar{S}}_{i j}
$$

and $L_{i j}^{a}$ is the anisotropic part of $L_{i j}$. We introduce an spatial averaging operation $\langle\cdot\rangle$ to avoid numerical problem. The specification of the average is problem dependent, as the universal definition of an ensemble average is inconvenient to use.

Applying this average to (A.58) and using least squares in the resulting equations leads to the formula

$$
C_{S}=\frac{\left\langle\sum L_{i j}^{a} M_{i j}\right\rangle}{\left\langle\sum M_{i j} M_{i j}\right\rangle} .
$$




\section{A.5 Code structure for incompressible fluid flow in the Fron- Tier package}

For the purpose of coupling different coordinate systems within a single code framework, we designed a structure for the solver part in the incompressible code using a $\mathrm{C}++$ class hierarchy structure. The benefit for using $\mathrm{C}++$ programming language is that we can derive the classes for specific coordinate systems from the base class so that most of the operations, such as initialization of the mesh and various utility functions, can be reused. For those functions which are related to different curvilinear coordinate systems, virtual functions are used in the base class and are specified in each specific class. Currently supported are classes for rectangular and cylindrical coordinates. Future plans call for a general curvilinear class as well. When using this hierarchy class structure, the concept of polymorphism in $\mathrm{C}++$ is used so that a specific function for different coordinate systems can be chosen at run time with the base class as the parameter for the driver of the entire solver.

The upper-level class Incompress_Solver_Basis is a pure virtual class which works as an interface for different interface methods which might have a totally different code structure including different variables. For example, we derive Incompress_Solver_Smooth_Basis and Incompress_Solver_EBM_Basis from it to define the solver for the Immersed Boundary Method and Embedded Boundary Method respectively. We focus on the IB Method here since it is in current use for the mixing flow problem. The Incompress_Solver_Smooth_Basis has all the variables required and all functions related to the initialization of the mesh and the indices for formation of the matrices in the diffusion and projection steps, which lead to a call to PETSc. All other functions are pure virtual functions which means they must be rewritten in the derived classes. The two most important pure virtual functions in this base class are solve() and setInitialCondition(), which work as an interface for the users to introduce their own solver for the incompressible Navier-Stokes equations and their own initialization of the velocity field. From the smooth base class, we derive the base class for 2D and 3D solver named Solver_Smooth_2D_Basis and Solver_Smooth_3D_Basis. These two classes define some basic operations, such as the calculation of Heaviside and Delta functions which differ between 2D and 3D. Then we derive the classes for different coordinate systems Solver_Smooth_2D_Cartesian, Solver_Smooth_3D_Cartesian and Solver_Smooth_3D_Cylindrical. These classes have different implementations of the virtual functions used in solve() so that we employ different incompressible NavierStokes equations for different coordinate systems.

Based on the different classes for different coordinate system, we also define a debugging class and rewrite the function which is related to the boundary conditions for using an exact solution as part of the order of convergence accuracy test. In summary, the graph for the class hierarchy is 


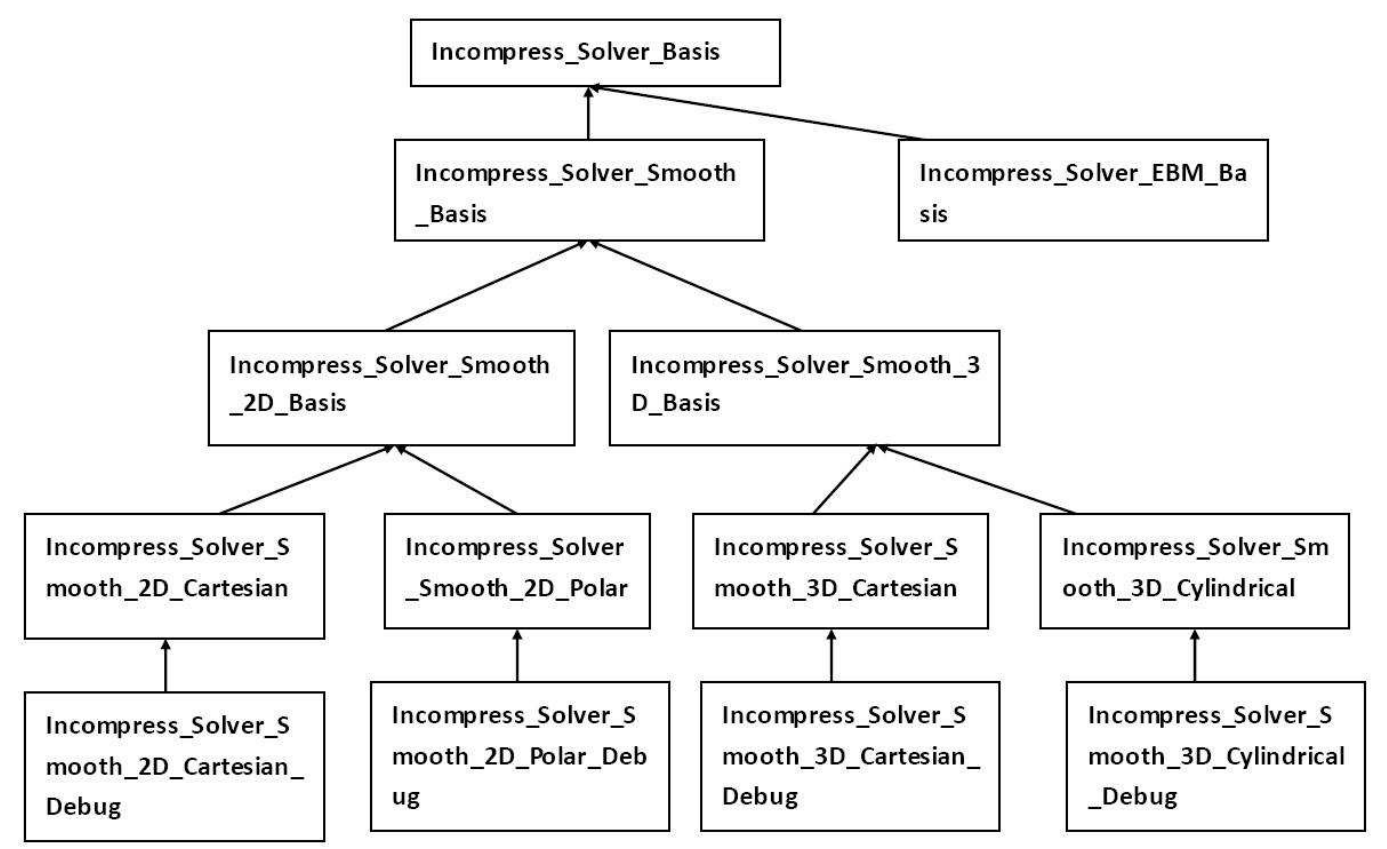

Fig. A.2: Hierarchy class structure for the incompressible solver.

\section{References}

Gretchen Baier. Liquid-Liquid extraction based on a new flow pattern: two-fluid Taylor-Couette flow. PhD thesis, University of Wisconsin-Madison, 1999.

Gretchen Baier and Michael D. Graham. Two-fluid Taylor-Couette flow: Experiments and linear theory for immiscible liquids between corotating cylinders. Phy. Fluids, 10:3045-3055, 1998.

Gretchen Baier and Michael D. Graham. Two-fluid Taylor-Couette flow with countercurrent axial flow: Linear theory for immiscible liquids between corotating cylinders. Phy. Fluids, 12:294-303, 2000.

Satish Balay, Kris Buschelman, Victor Eijkhout, William D. Gropp, Dinesh Kaushik, Matthew G. Knepley, Lois Curfman McInnes, Barry F. Smith, and Hong Zhang. PETSc users manual. Technical Report ANL-95/11 - Revision 3.0.0, Argonne National Laboratory, 2008.

M. Barad, P. Colella, D. T. Graves, P. Schwartz, B. van Straalen, and D. Trebotich. A Cartesian grid embedded boundary method for the incompressible Navier-Stokes equations. preprint submitted to Elsevier Science, 2009. 
J. B. Bell, P. Colella, and H. M. Glaz. A second order projection method for the incompressible Navier-Stokes equations. J. Comput. Phys., 85:257, 1989.

J. B. Bell, P. Colella, and H. M. Glaz. An efficient sceond-order projection method for viscous incompressible flow. Proceedings of the Tenth AIAA Computational Fluid Dynamics Conference, AIAA:360, 1991.

W. Bo, X. Liu, J. Glimm, and X. Li. A robust front tracking method: Verification and application to simulation of the primary breakup of a liquid jet. SIAM J. Sci. Comput., Accepted for publication, 2011.

J. U. Brackbill, D. B. Kothe, and C. Zemach. A continuum method for modeling surface tension. J. Comput. Phys., 100:335-354, 1992.

D. Brown, R. Cortez, and M. Minion. Accurate projection method for the incompressible Navier Stokes equations. J. Comput. Phys., 168:464-499, 2001.

S. Chandrasekhar. Hydrodynamic and Hydromagnetic Stability. Oxford University Press, Oxford, 1961.

A. J. Chorin. Numerical solution of the Navier Stokes equations. Math. Comp, 22: 745-762, 1968.

A. J. Chorin. On the convergence of discrete approximations to the Navier-Stokes equations. Math. Comp, 23:341, 1969.

P. Colella, D. T. Graves, B. J. Keen, and D. Modiano. A Cartesian grid embedded boundary method for hyperbolic conservasion laws. J. Comput. Phys., 211:347-366, 2006.

A. Davey. The growth of Taylor vortices in flow between rotating cylinders. J. Fluid Mech., 14:336-368, 1962.

S. Dong. Direct numercial simulation of turbulent Taylor-Couette flow. J. Fluid Mech., 587:373-393, 2007.

Jian Du, Brian Fix, James Glimm, Xicheng Jia, Xiaolin Li, Yunhua Li, and Lingling Wu. A simple package for front tracking. J. Comput. Phys., 213:613-628, 2006.

C. R. Ethier and D. A. Steinman. Exact fully 3d Navier-Stokes solutions for benchmarking. Int. J. Numer. Meth. Fl., 19:369-375, 1994.

R. P. Fedkiw, T. Aslam, B. Merriman, and S. Osher. A non-oscillatory Eulerian approach to interfaces in multimaterial flows (the ghost fluid method). J. Comput. Phys., 152:457-492, 1999. 
M. Germano, U. Piomelli, P. Moin, and W. H. Cabot. A dynamic subgrid scale eddy viscosity model. Phys. Fluids A, 3:1760-1765, 1991.

J. Glimm, M. J. Graham, J. W. Grove, X.-L. Li, T. M. Smith, D. Tan, F. Tangerman, and Q. Zhang. Front tracking in two and three dimensions. Comput. Math. Appl., 35(7):1-11, 1998a.

J. Glimm, J. W. Grove, X.-L. Li, K.-M. Shyue, Q. Zhang, and Y. Zeng. Three dimensional front tracking. SIAM J. Sci. Comput., 19:703-727, 1998b.

J. Glimm, J. W. Grove, X.-L. Li, and D. C. Tan. Robust computational algorithms for dynamic interface tracking in three dimensions. SIAM J. Sci. Comput., 21: 2240-2256, 2000.

C. Hirt and B. Nichols. Volume of fluid (VOF) method for the dynamics of free boundaries. J. Comput. Phys., 39:201-225, 1981.

Kazufumi Ito and Zhilin Li. Interface conditions for Stokes equations with a discontinuous viscosity and surface sources. Applied Mathematics Letters, 19:229-234, 2006.

Didier Jamet, David Torres, and J. U. Brackbill. On the theory and computation of surface tension: the elimination of parasitic currents through energy conservation in the second-gradient method. J. Comput. Phys., 182:262-76, 2002.

X. Jiao and H. Zha. Consistent computation of first- and second-order differential quantities for surface meshes. In Proceedings of the ACM Solid and Physical Modeling Symposium, pages 159-170, 2008.

H. Johansen and P. Colella. A Cartesian grid embedded boundary method for Poisson's equation on irregular domains. J. Comput. Phys, 147:60-85, 1998.

J. Van Kan. A second-order accurate pressure-correction scheme for viscous incompressible flow. SIAM J. Sci. Comput., 7:870, 1986.

M. Kang, R. P. Fedkiw, and X.-D. Liu. A boundary condition capturing method for multiphase incompressible flow. J. Sci. Comput., 15:323-360, 2000.

J. Kim and P. Moin. Application of a fractional-step method to incompressible NavierStokes equations. J. Comput. Phys., 59:308, 1985.

Youngsam Kim and Charles S. Peskin. Penalty immersed boundary method for an elastic boundary with mass. Phys. Fluids, 19:5], 2007. 
M. Kupiainen and Bjorn Sjogreen. A Cartesian embedded boundary method for the compressible Navier-Stokes equations. SIAM J. Sci. Comput., 41:94-117, 2009.

Ming-Chin Lai and Zhilin Li. A remark on jump conditions for the three-dimensional Navier-Stokes equations involving an immersed moving membrane. Applied Mathematics Letters, 14:149-154, 2001.

R. J. LeVeque and Z.-L. Li. The immersed interface method for elliptic equations with discontinuous coefficients and singular sources. SIAM J. Num. Anal., 31: 1019-1044, 1994.

Z. Li and K. Ito. The Immersed Interface Method: Numerical Solutions of PDEs Involving Interfaces and Irregular Domains. Frontiers Appl. Math. 33, SIAM, Piladelphia, 2006.

Zhilin Li. An overview of the immersed interface method and its applications. Taiwanese J. Mathematics, 7:1-49, 2003.

H. Lim, J. Iwerks, Y. Yu, J. Glimm, and D. H. Sharp. Verification and validation of a method for the simulation of turbulent mixing. Physica Scripta, T142:014014, 2010. Stony Brook Preprint SUNYSB-AMS-09-07 and Los Alamos National Laboratory preprint number LA-UR 09-07240.

X.-D. Liu, R. P. Fedkiw, and M. Kang. A boundary condition capturing method for Poisson's equation on irregular domains. J. Comput. Phys., 160:151-178, 2000.

X.-F. Liu, E. George, W. Bo, and J. Glimm. Turbulent mixing with physical mass diffusion. Phys. Rev. E, 73:056301, 2006.

X.-F. Liu, Y.-H. Li, J. Glimm, and X.-L. Li. A front tracking algorithm for limited mass diffusion. J. Comput. Phys., 222:644-653, 2007. Stony Brook University preprint number SUNYSB-AMS-06-01.

Richard Lueptow. Taylor-Couette flow. Scholarpedia, 4(11):6389, 2009.

T. Ma. Large eddy simulation of variable density flows. PhD thesis, University of Maryland, 2006.

P. McCorquodale, P. Colella, and H. Johansen. A Cartesian grid embedded boundary method for the heat equation on irregular domains. J. Comput. Phys., 273:620-635, 2001.

R. Mittal and G. Iaccarino. Immersed boundary methods. Annu. Rev. Fluid Mech., $37: 239-261,2005$. 
Zhou, Ray, Lim, Wang, de Almeida, Glimm, Li, and Jiao

C. S. Peskin. Numerical analysis of blood-flow in heart. J. Comput. Phys., 25:220-252, 1977.

C. S. Peskin. The immersed boundary method. Acta Numer., 11:479-517, 2002.

Stéphane Popinet and Stéphane Zaleski. A front-tracking algorithm for accurate representation of surface tension. Int. J. Numer. Meth. Fluids, 30:775-93, 1999.

R. C. Di Prima and Harry L. Swinney. Instabilities and transition in flow between concentric rotating cylinders. Topics in Applied Physics, 45:139-180, 1985.

P. H. Roberts. The solution of the characteristic value problems. Proc. R. Soc., 283: 550-556, 1965.

R. Scardovelli and S. Zaleski. Direct numerical simulation of free-surface and interfacial flow. Ann. Rev. Fluid Mech., 31:567-603, 1999.

P. Schwartz, M. Barad, P. Colella, and T. Ligocki. A Cartesian grid embedded boundary method for the heat equation and Poisson's equation in three dimensions. J. Comput. Phys., 211:531-550, 2006.

L. E. Scriven. Dynamics of a fluid interface: Equation of motion for newtonian surface fluids. Chem. Eng. Sci., 19:98-108, 1960.

J. A. Sethian. Level Set Methods. Cambridge University Press, 1996.

G. P. Smith and A. A. Townsend. Turbulent Couette flow between concentric cylinders at large Taylor numbers. J. Fluid Mech., 123:187-217, 1983.

E. M. Sparrow, W. D. Munro, and V. K. Jonsson. Instability of the flow between rotating cylinders:the wide gap problem. J. Fluid Mech., 20:35-46, 1974.

M. Sussman, P. Smereka, and S. Osher. A level set approach for computing solutions to incompressible two-phase flow. J. Comput. Phys., 77:146-154, 1994.

Zhijun Tan, D. V. Le, Zhilin Li, K. M. Lim, and B. C. Khoo. An immersed interface method for solving incompressible viscous flows with piecewise constant viscosity across a moving elastic membrane. J. Comput. Phys., 227:9955-9983, 2008.

Zhijun Tan, D. V. Le, K. M. Lim, and B. C. Khoo. An immersed interface method for the incompressible Navier-Stokes equations with discontinuous viscosity across the interface. SIAM J. Sci. Comput., 31:1798-1819, 2009.

G. I. Taylor. Stability of a viscous liquid contained between two rotating cylinders. Philos. Trans. R. Soc. London, Ser. A, 223:289-343, 1923. 
H. Terashima and G. Tryggvason. A front-tracking/ghost-fluid method for fluid interface in compressible flows. J. Comput. Phys., 228:4012-4037, 2009.

G. Tryggvason, B. Bunner, A. Esmaeeli, D. Juric, N. Al-Rawahi, W. Tauber, J. Han, S. Nas, and Y.-J. Jan. A front-tracking method for the computations of multiphase flow. J. Comput. Phys., 169:708-759, 2001.

S. O. Unverdi and G. Tryggvason. A front-tracking method for viscous, imcompressible, multi-fluid flows. J. Comput. Phys., 100(1):25-37, 1992.

S. Vedantam, J. B. Joshi, and S. B. Koganti. Three-dimensional CFD simulation of stratified two-fluid Taylor-Couette flow. Can. J. Chem. Eng., 84:279-288, 2006.

J. Walowit, S. Tsao, and R. C. DiPrima. Stability of flow between arbitrarily spaced concentric cylindrical sur faces including the effect of a radial temperature gradient. J. Appl. Mech., 31:585-593, 1964.

Shuqiang Wang, Roman V Samulyak, and Tongfei Guo. An embedded boundary method for elliptic and parabolic problems with interfaces and application to multimaterial systems with phase transitions. Acta Mathematica Scientia, 30B(2):499521,2010 .

S. T. Wereley and R. M. Lueptow. Azimuthal velocity in supercritical circular couette. Exp. Fluids, 18:1-9, 1994.

X. Ye, S. Cui, V. F. de Almeida, B. P. Hay, and B. Khomami. Uranyl nitrate complex extraction into TBP/dodecane organic solutions: a molecular dynamics study. Phys. Chem. Chem. Phys., 12:15406-9, 2010. 